\title{
DIRECTED GRAPHS AND TRAVELING WAVES
}

\author{
BY
}

\author{
DAVID TERMAN ${ }^{1}$
}

\begin{abstract}
The existence of traveling wave solutions for equations of the form $u_{t}=u_{x x}+F^{\prime}(u)$ is considered. All that is assumed about $F$ is that it is sufficiently smooth, $\lim _{|u| \rightarrow \infty} F(u)=-\infty, F$ has only a finite number of critical points, each of which is nondegenerate, and if $A$ and $B$ are distinct critical points of $F$, then $F(A) \neq F(B)$. The results demonstrate that, for a given function $F$, there may exist zero, exactly one, a finite number, or an infinite number of waves which connect two fixed, stable rest points. The main technique is to identify the phase planes, which arise naturally from the problem, with an array of integers. While the phase planes may be very complicated, the arrays of integers are always quite simple to analyze. Using the arrays of integers one is able to construct a directed graph; each path in the directed graph indicates a possible ordering, starting with the fastest, of which waves must exist. For a large class of functions $F$ one is then able to use the directed graphs in order to determine how many waves connect two stable rest points.
\end{abstract}

1. Introduction. This paper is concerned with proving the existence of traveling wave solutions of the equation

$$
u_{t}=u_{x x}+f(u)
$$

This equation arises in various branches of mathematical biology including population genetics, ecology and nerve conduction (see [1]). For most of the paper it will be more convenient to assume that $f(u)=F^{\prime}(u)$. All that we assume about $F$ is that:

(1.2) (c) $F$ has only a finite number of critical points,

(d) every critical point of $F$ is nondegenerate, and

(e) if $A$ and $B$ are distinct critical points of $F$, then $F(A) \neq F(B)$.

By a traveling wave solution of (1.1) we mean a nonconstant, bounded solution of the form $u(x, t)=U(z), z=x+\theta t$. These correspond to solutions which travel

\footnotetext{
Received by the editors August 31, 1984. Portions of this paper were presented at a conference sponsored by the American Mathematical Society at the University of New Hampshire, June 20-26, 1982.

1980 Mathematics Subject Classification. Primary 35K55.

Key words and phrases. Reaction-diffusion equation, traveling wave solution, directed graphs.

${ }^{1}$ Sponsored by the United States Army under Contract No. DAAG29-80-C-0041. This material is based upon work supported by the National Science Foundation under Grant No. MCS-7927062, Mod. 2. 
with constant shape and velocity, the velocity being $\theta$. If $U(z)$ is a traveling wave solution of (1.1), then $U$ satisfies the first order system of ordinary differential equations

$$
U^{\prime}=V, \quad V^{\prime}=\theta V-F^{\prime}(U) .
$$

For boundary conditions we assume that

$$
\lim _{z \rightarrow-\infty}(U, V)=(A, 0) \text { and } \lim _{z \rightarrow+\infty}(U, V)=(B, 0),
$$

where $A$ and $B$ are values where $F$ assumes a local maximum. We are interested in these boundary conditions because the values where $F$ assumes a local maximum correspond to the stable rest points of the partial differential equation (1.1). The traveling waves which connect stable rest points are, therefore, the ones of more physical interest. It now becomes convenient to make the following definition.

Definition. If $(U, V)$ is a solution of (1.3) and (1.4), then $U$ is an $A \rightarrow B$ connection.

We assume throughout this paper that the speed $\theta$ is positive. This simply means that we consider only waves which move to the left. Note that if $U(x, t)$ is a traveling wave solution moving to the left with speed $\theta$, then $U(-x, t)$ is a traveling wave solution moving to the right with speed $-\theta$.

The assumption $\theta>0$ implies that if $U$ is an $A \rightarrow B$ connection, then $F(A)<$ $F(B)$. To see why this is true consider the function

$$
H(U, V)=V^{2} / 2+F(U) \text {. }
$$

Using (1.3) one finds that

$$
d H(U, V) / d z=\theta V^{2} .
$$

Hence, $\theta>0$ implies that $H$ is increasing on solutions of (1.3). When $z=-\infty$, $H(U, V)=F(A)$, while, when $z=+\infty, H(U, V)=F(B)$. Hence, $F(A)<F(B)$.

The case when $F(U)$ has exactly two local maxima has been considered by a number of authors (see [1] for references). In this case $F^{\prime}(U)$ has the familiar cubic shape. Some work on the multistable case has been done by Fife and McLeod [2]. Fife and McLeod also considered the stability of traveling wave solutions. They showed that the monotone waves which connect stable rest points are stable. Hagan [3] also considered the question of stability. He proved that the nonmonotone waves which connect stable rest points are unstable.

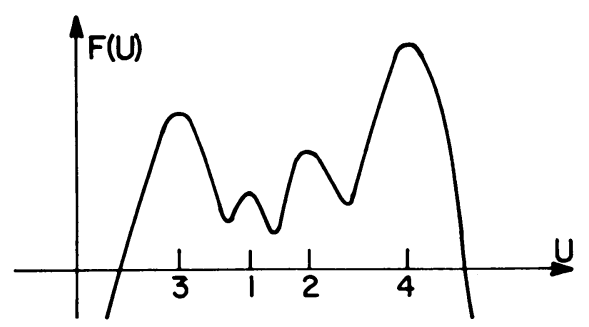

FIGURE 1. Notice that the local maxima of $F$ are ordered according to the height determined by $F$. 
The $(U, V)$ phase plane is the natural place to study the solutions of system (1.3). In the phase plane, the local maxima of $F$ correspond to saddles, while traveling wave solutions correspond to trajectories which "connect" the saddles. One can only expect saddle-saddle connections to exist for special values of the speed $\theta$. One of the difficulties in proving the existence of traveling wave solutions is that one does not know, a priori, what the speeds of the waves are.

The problem with phase plane analysis is that the phase planes become much too complicated for a general function $F$. To illustrate the approach we take, let $F$ be as shown in Figure 1. We suppose that there are four values of $U$, say $A, B, C$ and $D$, where $F$ assumes a local maximum. Assume that $A<B<C<D$ and $F(D)>F(A)$ $>F(C)>F(B)$. Notice that in Figure 1, we ordered the critical points according to the height determined by $F$. That is, we assign to the critical points $A, B, C$ and $D$, the integers 3,1, 2 and 4, respectively. Unless stated otherwise we always order the local maxima of $F$ in this manner. Our description of how many traveling waves exist shall be in terms of this ordering. Two functions which satisfy the conditions (1.2) are said to be in the same equivalence class if they have the same ordering of their local maxima. Hence, given a positive integer $n$, each permutation of the set $\{1,2, \ldots, n\}$ determines a unique equivalence class of functions.

The first step in analyzing the problem is to consider the phase plane when $\theta$ is very large, say $\theta=\theta_{0}$. If $\theta_{0}$ is sufficiently large, then the phase plane looks, qualitatively, like what is illustrated in Figure 2(A). As $\theta$ approaches $+\infty$ the unstable trajectories (those trajectories which approach a saddle in backwards time) become increasingly vertical, while the stable trajectories (those trajectories which approach a saddle in forwards time) become increasingly horizontal. These statements shall be made more precise in a later section. In Figure 2(B) we show the phase plane for $\theta=0$.

The next step is to start decreasing $\theta$ from $\theta_{0}$. As $\theta$ decreases, the phase plane changes in a continuous manner, at least until the first saddle-saddle connection is reached. Because we are starting from $\theta$ very large, the first saddle-saddle connection corresponds to the fastest traveling wave. There are different possibilities for what the fastest wave may be, depending upon the specific nature of the function $F$. However, by comparing the phase plane for $\theta=\theta_{0}$ with that for $\theta=0$ one is able to determine all the possibilities for the fastest wave. For a function in the equivalence class shown in Figure 1 , these possibilities are the $1 \rightarrow 3,1 \rightarrow 2$ and $2 \rightarrow 4$
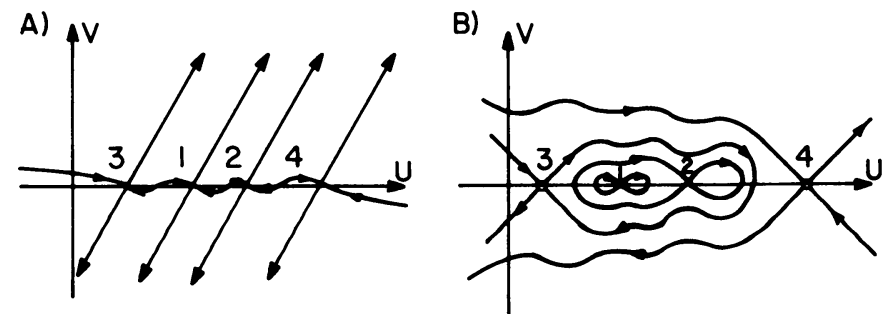

FIGURE 2. Phase planes for system (1.2), where $F$ is as shown in Figure 1. In (A), the speed $\theta$ is very large and, in (B), $\theta=0$. 
connections. Of course, which one of these waves is the fastest wave depends on the specific function $F$.

After the fastest connection has taken place, the qualitative features of the phase plane changes. This is shown in Figure 3. We now decrease $\theta$ further and determine, by comparing the new phase planes with the phase plane at $\theta=0$, all the possibilities for the second fastest wave. For example, if the fastest wave corresponds to a $1 \rightarrow 3$ connection, then the possibilities for the second fastest wave correspond to $2 \rightarrow 3,1 \rightarrow 2$ and $2 \rightarrow 4$ connections. A similar statement can be made if the fastest wave corresponds to either a $1 \rightarrow 2$ or $2 \rightarrow 4$ connection.

Continuing in this manner we construct a directed graph as shown in Figure 3. This graph describes all the possible orderings, starting with the fastest, of which connections can take place. Each particular function $F$ determines a path in the directed graph; the path indicates the fastest wave, second fastest wave, etc. Our immediate goal is to be able to construct and understand this directed graph.

One approach to constructing the directed graph would be to draw a lot of phase planes. This would be very tedious, if not impossible, because the phase planes become very complicated. Our approach is to assign to each phase plane an array of integers; the idea being that while the phase planes may be very complicated the arrays of integers will be quite simple. The arrays of integers will contain all the information needed about each phase plane in order to construct the directed graphs. Note that in order to construct a directed graph we must be able to do two things. First of all, given a particular phase plane, we must be able to determine all of the possibilities for the next fastest traveling wave. Hence, we need a scheme which tells us how to determine these possibilities by just considering the array of

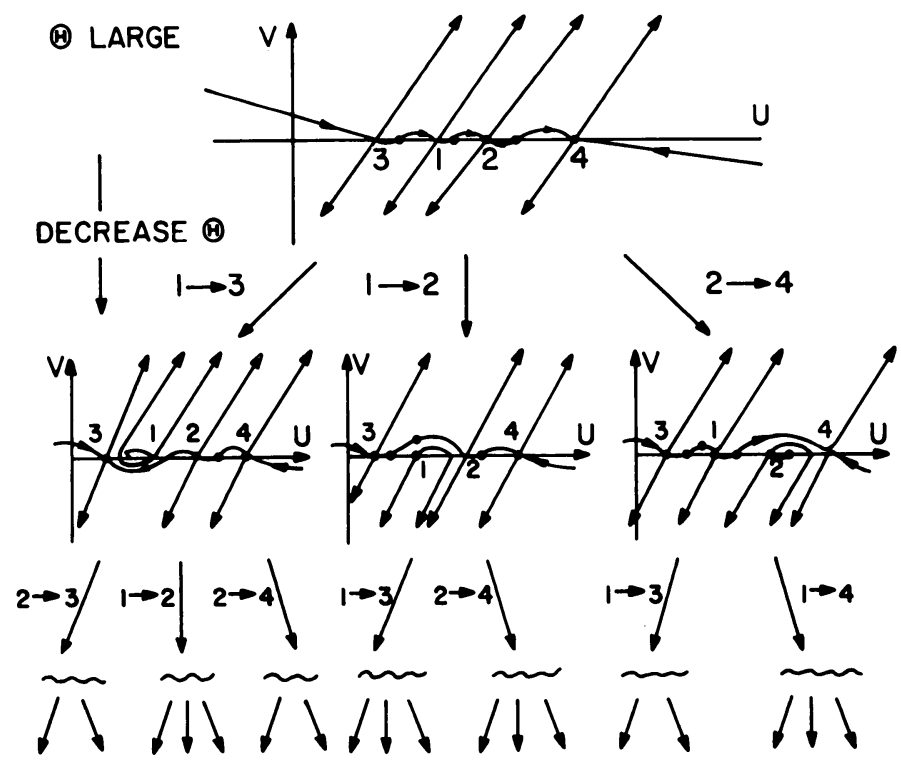

FIGURE 3. The directed graph for the equivalence class of functions shown in Figure 1. 
integers corresponding to the phase plane. Secondly, the qualitative features of the phase plane change after a particular connection has taken place. Hence, the array of integers might also change after a connection. Therefore, we need an algorithm which describes how to change the array of integers after a connection has occurred.

In the next section we show how to:

(a) assign an array of integers to each phase plane

(b) determine what the possibilities are for the next fastest wave by just considering the array of integers, and

(c) change the array of integers after a connection has taken place.

These operations are described in a series of propositions. Preliminary results needed for the proofs of the propositions are given in \$3. The actual proofs are given in $\$ 4$. In $\$ 5$ we shall see that the directed graphs may be very complicated. A technique is developed to simplify the graphs so that the task of determining how many waves exist becomes much easier. Finally, in $\$ 6$ we demonstrate how the directed graphs are used to determine the number of connections between two fixed critical points. We state explicitly for which functions $F$ the directed graphs may be used to determine whether zero, exactly one, a finite number, or an infinite number of connections exist between two critical points.

Before proceeding we introduce some notation. By a critical point we shall always mean a value of $U$ at which $F$ assumes a local maximum. The letters $A, B, C, D$ and $E$ will always be critical points. We let $\bar{A}=(A, 0)$. This denotes the saddle in the phase plane corresponding to the critical point $A$. Recall that the critical points of $F$ are ordered according to the height determined by $F$. Hence, to each critical point there corresponds an integer. We denote the integer corresponding to $A$ by $A^{*}$.

To each saddle $\bar{A}$, and $\theta \geqslant 0$ there corresponds two unstable trajectories. We denote these trajectories by $A_{N E}(z, \theta)$ and $A_{S W}(z, \theta)$. To emphasize the $U$ and $V$ coordinates of these trajectories we let

$$
A_{N E}(z, \theta)=\left(A_{N E}^{1}(z, \theta), A_{N E}^{2}(z, \theta)\right)
$$

and

$$
A_{S W}(z, \theta)=\left(A_{S W}^{1}(z, \theta), A_{S W}^{2}(z, \theta)\right) .
$$

$A_{N E}(z, \theta)$ has the property that $\lim _{z \rightarrow-\infty} A_{N E}(z, \theta)=\bar{A}$ and, for $z$ sufficiently negative, $A_{N E}^{1}(z, \theta)>A$ and $A_{N E}^{2}(z, \theta)>0 . A_{S W}(z, \theta)$ has the property that $\lim _{z \rightarrow-\infty} A_{S W}(z, \theta)=\bar{A}$ and, for $z$ sufficiently negative, $A_{S W}^{1}(z, \theta)<A$ and $A_{S W}^{2}(z, \theta)<0$.

We denote the stable trajectories of $\bar{A}$ by $A_{N W}(z, \theta)$ and $A_{S W}(z, \theta)$. These have the property that

$$
\lim _{z \rightarrow+\infty} A_{N W}(z, \theta)=\bar{A}=\lim _{z \rightarrow+\infty} A_{S W}(z, \theta)
$$

and, for $z$ sufficiently large, $A_{N W}^{1}(z, \theta)<A, A_{N W}^{2}(z, \theta)>0, A_{S E}^{1}(z, \theta)>A$ and $A_{S E}^{2}(z, \theta)<0$. 
2. Rules for constructing the directed graphs. The simplest way to illustrate how to perform the operations described in (1.7) is with a specific example. Suppose that $F$ is in the equivaience class of functions illustrated in Figure 1 , and when $\theta=\theta_{0}$ the phase plane is as shown in Figure 4. Only the unstable trajectories are shown in Figure 4 because, in order to perform the operations described in (1.7), that is all we need to know. As usual we have ordered the saddles according to the height determined by $F$.

We now describe how to construct the array of integers corresponding to the phase plane shown in Figure 4. The first step is to draw a big rectangle $\mathscr{R}$ around all of the rest points. In the next section we show that $\mathscr{R}$ can be chosen so that all of the connections, for all values of $\theta$, lie inside of it. It is also shown in the next section that $\mathscr{R}$ can be chosen so that the unstable trajectories can only leave $\mathscr{R}$ through either its top or bottom sides. The next step in assigning an array of integers to the phase plane is to locate those points on the top and bottom sides of $\mathscr{R}$ which lie on one of the unstable trajectories. It is possible that one of the unstable trajectories crosses the boundary of $\mathscr{R}$ more than once. We only consider those points on the boundary of $\mathscr{R}$ which correspond to the first time that an unstable trajectory leaves $\mathscr{R}$. To each one of these points there corresponds an integer; the integer corresponds to the saddle on whose unstable trajectory the point lies. We now have two lists of integers. One is associated with the top side of $\mathscr{R}$, and the other to the bottom side. For the example shown in Figure 4 the two lists are $\{3,1,4\}$ and $\{3,2,2,1,4\}$. Combining these two lists we obtain

$$
\frac{314}{32214}
$$

This is the desired array of integers!

The following two propositions demonstrate how one determines, from the array of integers, the possibilities for the next fastest traveling wave solution, and how this information is used to conclude the existence of traveling wave solutions. Proposition 2.1 states that each possibility for the next fastest wave must occur, although not necessarily as the next wave. This result restricts the possible paths that can be taken for a given $F(U)$.

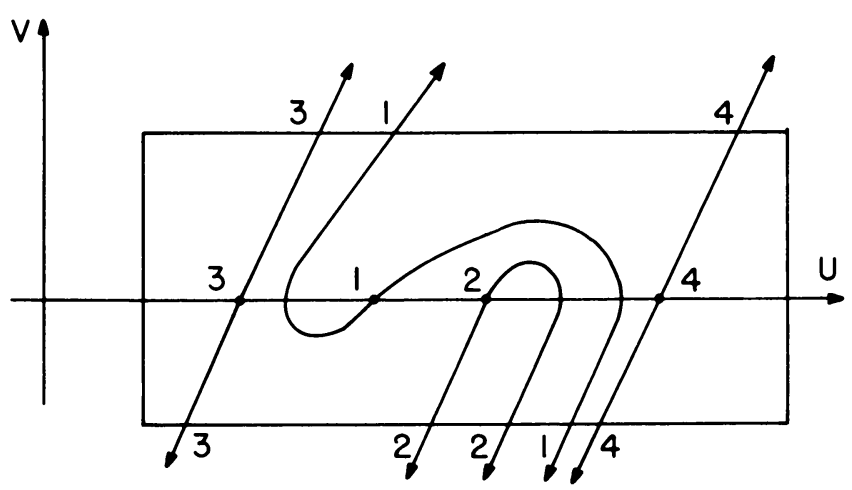

FIGURE 4. The array of numbers associated with this phase plane is $\frac{314}{32214}$. 
Proposition 2.1. Suppose that the array corresponding to a given phase plane, which occurs with speed $\theta_{0}$, is

$$
\frac{T_{1} T_{2} \cdots T_{m}}{B_{1} B_{2} \cdots B_{n}}
$$

If for some $k, T_{k}<T_{k+1}$, then there exists a $T_{k} \rightarrow T_{k+1}$ connection for some $\theta<\theta_{0}$. If for some $k, B_{k}>B_{k+1}$, then there exists a $B_{k+1} \rightarrow B_{k}$ connection for some $\theta<\theta_{0}$.

Proposition 2.2. The connections described in Proposition 1.1 are all of the possibilities for the next fastest wave. That is, suppose that there exists an $A \rightarrow B$ connection with speed $\theta_{1}$ such that $\theta_{1}<\theta_{0}$, and there do not exist any connections with speed $\theta \in\left(\theta_{1}, \theta_{0}\right)$. Then there exists an integer $k$ such that either $A^{*}=T_{k}$ and $B^{*}=T_{k+1}$, or $A^{*}=B_{k+1}$ and $B^{*}=B_{k}$.

Let us apply these two propositions to the phase plane shown in Figure 4. Considering the array (2.1) we find that the possibilities for the next fastest wave correspond to $1 \rightarrow 4,2 \rightarrow 3$ and $1 \rightarrow 2$ connections. Note that a $1 \rightarrow 3$ connection may or may not exist for some $\theta \in\left(0, \theta_{0}\right)$, but it cannot correspond to the next fastest wave. Furthermore, $1 \rightarrow 4,2 \rightarrow 3$ and $1 \rightarrow 2$ connections must all exist for some speeds less than $\theta_{0}$.

We now need an algorithm which tells us how the array changes after a connection has taken place. This algorithm is described in the next proposition. For this proposition we assume that the array is known for some value of the speed, say $\theta=\theta_{0}$. We also assume that there exists $\theta_{1}<\theta_{0}$ such that no connections exist with speed $\theta \in\left(\theta_{1}, \theta_{0}\right)$, and an $A \rightarrow B$ connection exists with speed $\theta_{1}$. For now, we assume that there is only one connection with speed $\theta_{1}$. After the proof of Proposition 2.3 in subsection 4(B) we discuss what happens if there exists more than one wave with the same speed. Note that there must be two $B$ 's in the array since to each saddle there corresponds two unstable directions. Of course, there are two $A$ 's also, but the 'other $A$ ' will play no role. In the proposition we consider two cases depending on whether the 'other $B$ ' is on the top or the bottom of the array.

Proposition 2.3. If the other $B$ is on the top, then after the $A \rightarrow B$ connection everything in the array remains exactly the same except that the $A$ is moved to the immediate right of the other $B$. If the other $B$ is on the bottom, then after the $A \rightarrow B$ connection everything in the array remains exactly the same except that the $A$ is moved to the immediate left of the other $B$.

Here are two examples of what may happen:

$$
\begin{aligned}
& \frac{\cdots A B \cdots B \cdots}{\cdots} \stackrel{A \rightarrow B}{\rightarrow} \frac{\cdots B \cdots B A \cdots}{\cdots} \\
& \frac{\cdots A B \cdots}{\cdots B \cdots} \stackrel{A \rightarrow B}{\rightarrow} \frac{\cdots B \cdots}{\cdots A B \cdots} .
\end{aligned}
$$


Applying this proposition to the phase plane shown in Figure 4 we obtain the following portion of the graph:

$$
\begin{array}{ccc} 
& \frac{314}{32214} & \\
1 \rightarrow 4 \swarrow & \downarrow 2 \rightarrow 3 & \searrow 1 \rightarrow 2 \\
\frac{34}{322114} & \frac{3214}{3214} & \frac{314}{31224}
\end{array}
$$

This completes our description of how to perform the three operations described in (1.7). However, it remains to demonstrate how one begins the directed graph. That is, the directed graph is generated by a particular array. This array corresponds to the phase plane when the speed $\theta$ is very large. To determine this array we use the fact, which will be proved in the next section, that when $\theta$ is very large, the unstable trajectories are nearly vertical. Hence, if the ordering of the saddles is $A_{1}, A_{2}, \ldots, A_{n}$, then the generator of the graph is the array

$$
\frac{A_{1} A_{2} \cdots A_{n}}{A_{1} A_{2} \cdots A_{n}}
$$

For example, if $F$ is in the equivalence class of functions shown if Figure 1, then the graph starts with the array

$$
\frac{3124}{3124} \text {. }
$$

Propositions 2.1, 2.2 and 2.3 are proved in $\$ 4$. We now demonstrate, with a specific example, how the propositions are used to construct an entire directed graph, and how the graph is used to prove the existence of traveling wave solutions.

Consider a function $F$ which is in the equivalence class of functions shown in Figure 5.

Using the directed graph we prove the following theorem.

THEOREM 2.1. (a) There exists a unique $2 \rightarrow 3$ connection.

(b) There exists a finite number of $1 \rightarrow 3$ connections.

(c) There exists an infinite number of $1 \rightarrow 2$ connections.

By a finite (infinite) number of $A \rightarrow B$ connections we mean that there exists a finite (infinite) number of speeds $\theta$ for which there exists a solution of (1.3) and (1.4). In Proposition 2.4, it is shown that if there exists an infinite number of connections, then the speeds of these waves must satisfy $\lim _{k \rightarrow \infty} \theta_{k}=0$.

We assume throughout that Theorem 2.1(a) is true. The existence of a $2 \rightarrow 3$ connection can be proved using a simple shooting argument, comparing the phase

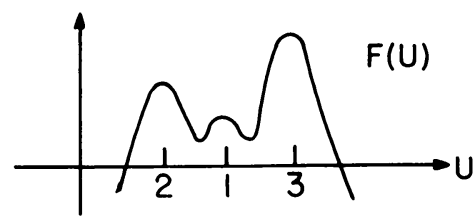

FIGURF 5. For the equivalence class of functions shown here there exists an infinite number of $1 \rightarrow 2$ connections, a finite number of $1 \rightarrow 3$ connections, and precisely one $2 \rightarrow 3$ connection. 
plane for $\theta$ very large with the phase plane when $\theta=0$. The existence and uniqueness of a $2 \rightarrow 3$ connection also follows from our later results.

To prove Theorem 2.1(b) and (c) we consider the graph corresponding to the equivalence class of functions shown in Figure 5. Note that the graph is generated by the array

$$
\frac{213}{213} \text {. }
$$

The rest of the directed graph is constructed using Propositions 2.1-2.3. It is shown in Figure 6.

Note that the function $F$ determines a path in the directed graph shown in Figure 6. What we know about the path so far is that it must start at the node

$$
\frac{213}{213}
$$

obey the arrows, and eventually cross an edge which corresponds to a $2 \rightarrow 3$ connection. Note that there is only one edge which corresponds to a $2 \rightarrow 3$ connection in the entire directed graph. Hence, the path corresponding to $F$ must eventually be at the array

$$
\frac{3}{21123} \text {. }
$$

Once the path reaches this array, its only choice is to go around the loop in Figure 6 corresponding to a $1 \rightarrow 2$ connection. According to Proposition 2.1 the path can never stop. It is forced to go around the $1 \rightarrow 2$ loop an infinite number of times. This implies that there must exist an infinite number of $1 \rightarrow 2$ connections. We shall see later that the number of $1 \rightarrow 2$ connections is countably infinite. If the speeds of these waves are $\left\{\theta_{k}\right\}$, then $\lim _{k \rightarrow \infty} \theta_{k}=0$. In fact, we shall prove the following proposition.

Proposition 2.4. For any $F$ which satisfies (1.2) and any $\theta_{0}>0$, there can exist at most a finite number of connections with speeds greater than $\theta_{0}$.

This last statement is used to prove Theorem 2.1(b). The $2 \rightarrow 3$ connection must occur with some finite speed, say $\theta_{1}$. There certainly are not any $1 \rightarrow 3$ connections

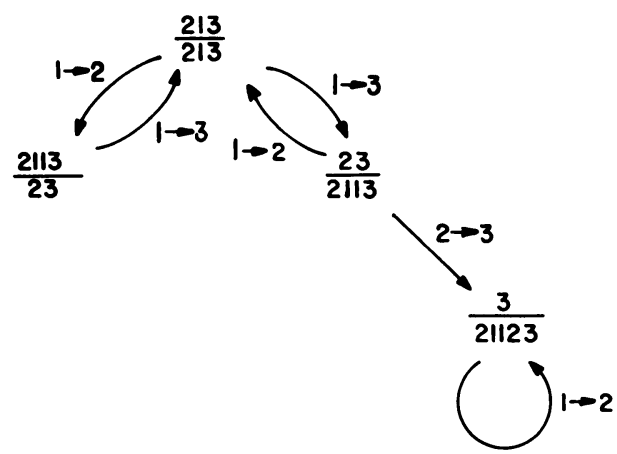

FIGURE 6. The directed graph associated with the equivalence class of functions shown in Figure 6. 
with speeds less than $\theta_{1}$ because then we are caught in the $1 \rightarrow 2$ loop. Since there are only a finite number of waves with speeds greater than $\theta_{1}$, there can exist at most a finite number of $1 \rightarrow 3$ connections.

\section{Preliminary results.}

(A) Phase plane for $\theta=0$. Recall from (1.5) and (1.6) that the function $H(U, V)=$ $V^{2} / 2+F(U)$ is constant on solutions when $\theta=0$. Therefore, if, on a particular trajectory, $H(U, V)=K$, then the curve in the phase plane traced out by the trajectory is explicitly given by the formula

$$
V= \pm\{2(K-F(U))\}^{1 / 2}
$$

Suppose that the critical points $A$ and $B$ satisfy $A<B$ and $F(A)<F(B)$. It then follows that $A_{N E}(z, 0)=A_{S E}(z, 0)$. In fact, (3A.1) shows that the top half of the trajectory is given by $V=+\{2(F(A)-F(U))\}^{1 / 2}$, while the bottom half is given by $V=-\{2(F(A)-F(U))\}^{1 / 2}$. Since $A_{N E}(z, 0)$ approaches $\bar{A}$ in both forwards and backwards time, we say that $A_{N E}(z, 0)$ is a homoclinic orbit. A similar analysis shows that if $A>B$ and $F(A)<F(B)$, then $A_{S W}(z, 0)=A_{N W}(z, 0)$.

Let us return to the case when $A<B$ and $F(A)<F(B)$. Let

$$
\beta=\inf \{U: U>A \text { and } F(U)=F(A)\} .
$$

Clearly $\beta<B$. Note that $A_{N E}(z, 0)$ intersects the $U$-axis at $U=\beta$. Furthermore, $A<A_{N E}(z, 0) \leqslant \beta$ for each $z \in \mathbf{R}$. This result follows from (3A.1).

Now suppose that there does not exist a critical point $C$ such that $A<C<B$ and $F(C)>F(B)$. We show that the homoclinic orbit $A_{N E}(z, 0)$ "lies between" $B_{N W}(z, 0)$ and $B_{S W}(z, 0)$. That is, given $\alpha \in(A, \beta)$, there exists four uniquely determined constants, $V_{1}, V_{2}, V_{3}$ and $V_{4}$, such that $V_{1}<V_{2}<0<V_{3}<V_{4},\left(\alpha, V_{1}\right)$ lies on $B_{S W}(z, 0),\left(\alpha, V_{2}\right)$ lies on $A_{S E}(z, 0),\left(\alpha, V_{3}\right)$ lies on $A_{N E}(z, 0)$, and $\left(\alpha, V_{4}\right)$ lies on $B_{N W}(z, 0)$. This result is proved by contradiction. Suppose, for example, there existed constants $\alpha, V_{3}$ and $V_{4}$ such that $A<\alpha \leqslant \beta, 0<V_{4}<V_{3},\left(\alpha, V_{3}\right)$ lies on $A_{N E}(z, 0)$, and $\left(\alpha, V_{4}\right)$ lies on $B_{N W}(z, 0)$. Since $H(U, V)$ is constant on solutions, it follows that

$$
F(A)=V_{3}^{2} / 2+F(\alpha) \text { and } F(B)=V_{4}^{2} / 2+F(\alpha) .
$$

This contradicts the assumption that $F(A)<F(B)$ and $V_{3}>V_{4}$. One must, of course, show that $B_{N W}(z, 0)$ and $B_{S W}(z, 0)$ both cross each line $U=\alpha, A<\alpha \leqslant \beta$, at a unique point. This follows from the explicit expression for the trajectories given by (3A.1) and the assumption that there does not exist a critical point $C$ such that $A<C<B$ and $F(C)>F(B)$.

Finally, let $D$ be the region enclosed by the homoclinic orbit $A_{N E}(z, 0)$. In $D$, $H(U, V)<F(A)$. It therefore follows from (1.5) that, for each $\theta>0, A_{N E}(z, \theta)$ lies outside $D$, while $A_{S W}(z, \theta)$ lies inside $D$.

(B) Phase plane for $\theta$ very large. Let $A$ be any local maximum of $F$. We show that as $\theta$ becomes very large the unstable trajectories $A_{N E}(z, \theta)$ and $A_{S W}(z, \theta)$ become increasingly vertical. More precisely, given $\varepsilon>0$, there exists a constant $\theta(\varepsilon)$ such 
that if $\theta>\theta(\varepsilon)$, then $A_{N E}(z, \theta)$ lies entirely inside the region $S^{+}=\{(U, V): A<U$ $<\varepsilon V+A\}$, and $A_{S W}(z, \theta)$ lies entirely inside the region $S^{-}=\{(U, V): \varepsilon V+A<U$ $<A\}$.

The first step is to linearize the system (1.3) about the saddle $\bar{A} \equiv(A, 0)$. If $F^{\prime \prime}(A)=-\alpha$, where $\alpha>0$, then the eigenvalues at $\bar{A}$ are

$$
\lambda^{ \pm}(\theta)=\left(\theta^{ \pm} \sqrt{\theta^{2}+4 \alpha}\right) / 2 .
$$

An eigenvector corresponding to $\lambda^{+}(\theta)$ is $\left(1, \lambda^{+}(\theta)\right)$. Therefore, $A_{N E}(z, \theta)$ and $A_{S W}(z, \theta)$ both approach $\bar{A}$, in negative time, tangent to the line through $(A, 0)$ with slope $\lambda^{+}(\theta)$. Since $\lim _{\theta \rightarrow+\infty} \lambda^{+}(\theta)=+\infty$, the claim is true near $\bar{A}$. To complete the proof we note that both $S^{+}$and $S^{-}$are positively invariant for $\theta$ sufficiently large. That is, every trajectory which lies inside $S^{+}$or $S^{-}$for some time $z_{0}$ must remain inside $S^{+}$or $S^{-}$, respectively, for $z>z_{0}$. This is proved by considering (1.3) and showing that at each point on the boundary of $S^{+}$or $S^{-}$, the vector field determined by the right-hand side of (1.3) points into $S^{+}$or $S^{-}$.

A similar argument shows that as $\theta$ becomes very large, the stable trajectories $A_{N W}(z, \theta)$ and $A_{S E}(z, \theta)$ become increasingly horizontal. One shows that, given $\varepsilon>0$, there exists a constant $\theta(\varepsilon)$ such that if $\theta>\theta(\varepsilon)$, then $A_{N W}(z, \theta)$ lies entirely inside the region $J^{+}=\{(U, V): 0<V<-\varepsilon(U-A)\}$, while $A_{S E}(z, \theta)$ lies entirely inside the region $J^{-}=\{(U, V):-\varepsilon(U-A)<V<0\}$. As before, one proves this is true near $\bar{A}$ by linearizing the system (1.3) at $\bar{A}$. One then shows that $J^{+}$and $J^{-}$are negatively invariant.

(C) The rectangle $\mathscr{R}$. Given $F$, we show how to construct the rectangle $\mathscr{R}$ so that the unstable trajectories can only leave $\mathscr{R}$ through its top or bottom sides. Furthermore, except for the connections, each unstable trajectory must leave $\mathscr{R}$.

Let $C$ be the critical point at which $F$ assumes its absolute maximum. Let $D$ and $E$ be the critical points which satisfy $D<A<E$ for all other critical points $A$. Set $D(z)=D_{S W}(z, 0)$ and $E(z)=E_{N E}(z, 0)$. In order to distinguish the $U$ and $V$ coordinates of these trajectories we suppose that $D(z)=\left(D_{1}(z), D_{2}(z)\right)$ and $E(z)=$ $\left(E_{1}(z), E_{2}(z)\right)$. Note that

$$
D_{N W}(z, 0)=\left(D_{1}(z),-D_{2}(z)\right) \text { and } E_{S E}(z, 0)=\left(E_{1}(z),-E_{2}(z)\right) .
$$

Furthermore, for all $z, D_{1}^{\prime}(z)<0, D_{2}^{\prime}(z)<0, E_{1}^{\prime}(z)>0, E_{2}^{\prime}(z)>0$,

$$
\lim _{z \rightarrow \infty} D_{1}(z)=\lim _{z \rightarrow \infty} D_{2}(z)=-\infty \text { and } \lim _{z \rightarrow \infty} E_{1}(z)=\lim _{z \rightarrow \infty} E_{2}(z)=\infty \text {. }
$$

These facts are simple consequences of our assumptions and the form of system (1.3).

Choose $V_{1}$ so that $V_{1}>0$ and $H\left(V_{1}, U\right)>F(C)$ for all $U \in(D, E)$. Let $R_{1}$ be equal to the value of $E_{1}(z)$ at the point where $E_{2}(z)=V_{1}$, and let $R_{2}$ be equal to the value of $D_{1}(z)$ at the point where $D_{2}(z)=-V_{1}$. Let $\mathscr{R}$ be the rectangle with corners at the points $\left(R_{1}, V_{1}\right),\left(R_{1},-V_{1}\right),\left(R_{2}, V_{1}\right)$ and $\left(R_{2},-V_{1}\right)$.

We now show that all of the unstable trajectories arising from the saddles can only leave $\mathscr{R}$ through its top or bottom sides. Note that on the trajectory $E_{N E}(z, \theta)$, $U^{\prime}(z)>0$ and $V^{\prime}(z)>0$. This is true for all $\theta \geqslant 0$. Furthermore, since $H(U, V)<$ $F(E)$ in the region $\left\{(U, V):-E_{2}(z)<V<E_{2}(z), U>E\right\},(1.6)$ implies that, for 
each $\theta, E_{N E}(z, \theta)$ leaves $\mathscr{R}$ on its top side. A similar analysis shows that, for each $\theta$, $D_{S W}(z, \theta)$ leaves $\mathscr{R}$ on its bottom side after traveling in a monotone decreasing fashion. Now, fix $\theta$ and let $T(z)$ be one of the unstable trajectories with corresponding speed $\theta$. Note that $E_{N E}(z, \theta)$ prevents $T(z)$ from leaving the right side of $\mathscr{R}$ with $V \geqslant 0$. Similarly, $D_{S W}(z, \theta)$ prevents $T(z)$ from leaving the left side of $\mathscr{R}$ with $V \leqslant 0$. On the other hand, $T(z)$ cannot exit the right side of $\mathscr{R}$ with $V \leqslant 0$ or the left side of $\mathscr{R}$ with $V \geqslant 0$, because, on those portions of the boundary, the vector field defined by the right-hand side of (1.3) points into $\mathscr{R}$. Therefore, $T(z)$ can only leave $\mathscr{R}$ through its top or bottom sides.

We now prove the following. Let $T(z)$ be one of the unstable trajectories which leaves $\mathscr{R}$, and let $T\left(z_{0}\right)=P$ be the point on the boundary of $\mathscr{R}$ where $T(z)$ first leaves $\mathscr{R}$. As usual, we set $T(z)=\left(T_{1}(z), T_{2}(z)\right)$. We show that $T_{2}(z) \neq 0$ for all $z>z_{0}$. Furthermore, if $P$ is on the top side of $\mathscr{R}$, then

$$
\lim _{z \rightarrow \infty} T_{1}(z)=\lim _{z \rightarrow \infty} T_{2}(z)=\infty,
$$

while if $P$ is on the bottom side of $\mathscr{R}$, then

$$
\lim _{z \rightarrow \infty} T_{1}(z)=\lim _{z \rightarrow \infty} T_{2}(z)=-\infty
$$

We suppose that $P$ is on the top side of $\mathscr{R}$ since the other case is similar. Let $P=\left(P_{1}, P_{2}\right)$.

Suppose that $T_{2}\left(z_{1}\right)=0$ for $z_{1}>z_{0}$, and $T_{2}(z)>0$ for $z \in\left(z_{0}, z_{1}\right)$. Since $U^{\prime}=V$ we have $T_{1}\left(z_{1}\right)>T_{1}\left(z_{0}\right)$. Let

$$
H\left(z_{0}\right)=H\left(T_{1}\left(z_{0}\right), T_{2}\left(z_{0}\right)\right) \text { and } H\left(z_{1}\right)=H\left(T_{1}\left(z_{1}\right), T_{2}\left(z_{1}\right)\right) \text {. }
$$

It follows from (1.6) that $H\left(z_{0}\right)<H\left(z_{1}\right)$. We show that this leads to a contradiction by considering a few cases. First of all note that it is impossible for $P_{1}<D$. If this were true, then, since $F^{\prime}(U)>0$ for $U<D$, (1.3) implies that $T_{1}(z) \rightarrow-\infty$ and $T_{2}(z) \rightarrow+\infty$ as $z \rightarrow-\infty$. This contradicts the assumption that $T(z)$ is one of the unstable trajectories.

Now suppose that $D<P_{1}<E$. From the way we chose $V_{1}$ it follows that $H\left(z_{0}\right)=V_{1}^{2} / 2+F\left(P_{1}\right) \geqslant F(C)$. On the other hand, $H\left(z_{1}\right)=F\left(T_{1}\left(z_{1}\right)\right)$. Since $F(C)$ $>F(U)$ for all $U$, we have a contradiction.

Finally, suppose that $P_{1}>E$. Then, since $F^{\prime}(U)<0$ for $U>E$ and $T_{1}\left(z_{1}\right)>$ $T_{1}\left(z_{0}\right)$, it follows that $F\left(T_{1}\left(z_{1}\right)\right)<F\left(T_{1}\left(z_{0}\right)\right)$. Hence, $H\left(z_{0}\right)=V_{1}^{2} / 2+F\left(T_{1}\left(z_{0}\right)\right)>$ $F\left(T_{1}\left(z_{1}\right)\right)=H\left(z_{1}\right)$. This, again, is a contradiction.

To conclude our discussion of $\mathscr{R}$ we must show that, for $\theta>0$, each unstable trajectory is either a connection or else leaves $\mathscr{R}$. This, however, is a consequence of (1.6).

(D) Simple but important results. The following results are needed a number of times in the proofs of Propositions 2.1-2.3. We assume throughout that $p$ and $q$ are two constants with $p<q$. For some $\theta>0$, let $P(z)$ and $Q(z)$ be the solutions starting at $(p, 0)$ and $(q, 0)$, respectively. We assume that

$$
P(z)=\left(P_{1}(z), P_{2}(z)\right) \text { and } Q(z)=\left(Q_{1}(z), Q_{2}(z)\right) \text {. }
$$


We also assume that $(p, 0)$ and $(q, 0)$ are not rest points, and, without loss of generality, $P(0)=(p, 0)$ and $Q(0)=(q, 0)$. Finally, we assume that $P(z)$ and $Q(z)$ leave $\mathscr{R}$ with $U$-coordinate equal to $\alpha$ and $\beta$, respectively.

Lemma 3D.1. Assume that $d P_{2}(0) / d z>0$ and the first place where $P(z)$ leaves $\mathscr{R}$ is through its top side. If $q-p$ is sufficiently small, then the first place where $Q(z)$ leaves $\mathscr{R}$ is through its top side, and $\alpha<\beta$.

ProOF. By making $q-p$ sufficiently small, we may assume that $P(z)$ and $Q(z)$ are as close as we please to each other as long as they are in $\mathscr{R}$. Therefore, if $q-p$ is sufficiently small, then $d Q_{2}(z) / d z>0$, and $Q(z)$ leaves $\mathscr{R}$ through its top side. To complete the proof we follow, in some detail, $P(z)$ and $Q(z)$ around in the phase plane. In what follows the reader should refer to Figure 7.

Choose $\left\{z_{j}\right\}, j=0,1, \ldots, n$, such that $z_{0}=0, z_{j}<z_{j+1}$ for each $j$, and $P_{2}(z)=0$ for $z \geqslant 0$ if and only if $z=z_{j}$ for some $j$. Let $\eta_{j}=P_{1}\left(z_{j}\right)$. Of course, it is possible that $n=0$. The above assumptions imply that $n$ is an even integer and

$$
\eta_{n}<\eta_{n-2}<\cdots<\eta_{2}<\eta_{0}<\eta_{1}<\eta_{3}<\cdots<\eta_{n-3}<\eta_{n-1} .
$$

Here we use the fact that $U^{\prime}=V$ which implies that the trajectories spiral in a clockwise direction. If $q-p$ is sufficiently small, then there must exist $\left\{\xi_{k}\right\}$, $k=0,1, \ldots, n$, such that $\xi_{0}=0, \xi_{j}<\xi_{j+1}$ for such $j$, and $Q_{2}(z)=0$ for $z \geqslant 0$ if and only if $z=z_{j}$ for some $j$. Let $\zeta_{j}=Q_{1}\left(\xi_{j}\right)$. By choosing $q-p$ small we can make $\sup _{0 \leqslant j \leqslant n}\left|\eta_{j}-\zeta_{j}\right|$ as small as we please. Furthermore, because $p<q$ and $d P_{2}(0) / d z$ $>0$, it follows that if $j$ is even, then $\eta_{j}<\zeta_{j}$. If $j$ is odd, then $\eta_{j}>\zeta_{j}$. Since $n$ is even, we have $\eta_{n}<\zeta_{n}$. Hence, from the times $P(z)$ and $Q(z)$ last cross the $U$-axis until the times they exit $\mathscr{R}, P(z)$ lies to the left of $Q(z)$. In particular, $\alpha<\beta$.

There are other cases to consider besides that described in Lemma 3D.1. Here we just list the results. The proofs of these results are almost identical to the one just given.

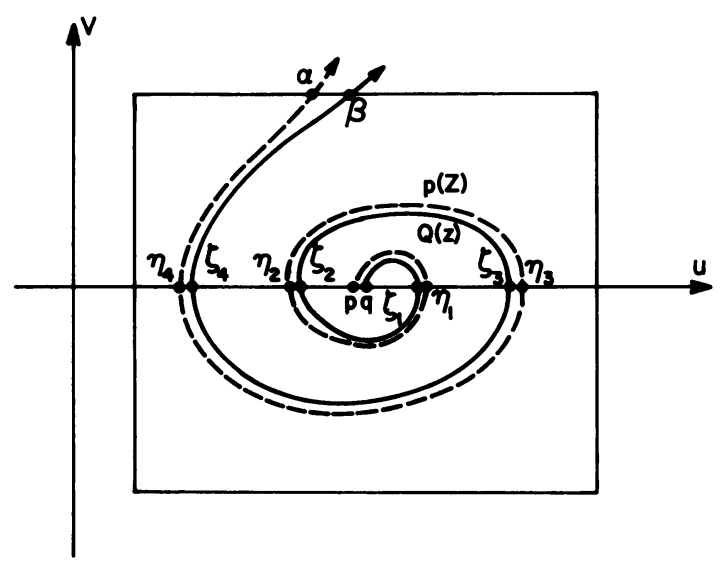

FIGURE 7. This is the situation described in Lemma 3D.1. The dotted curve is $P(z)$; the solid curve is $Q(z)$. 
Lemma 3D.2. Assume that $d P_{2}(0) / d z<0$ and $P(z)$ leaves $\mathscr{R}$ through its top side. If $q-p$ is sufficiently small, then $Q(z)$ leaves $\mathscr{R}$ through its top side and $\alpha>\beta$.

Lemma 3D.3. Assume that $d P_{2}(0) / d z>0$ and $P(z)$ leaves $\mathscr{R}$ through its bottom side. If $q-p$ is sufficiently small, then $Q(z)$ leaves $\mathscr{R}$ through its bottom side and $\alpha>\beta$.

Lemma 3D.4. Assume that $d P_{2}(0) / d z<0$ and $P(z)$ leaves $\mathscr{R}$ through its bottom side. If $q-p$ is sufficiently small, then $Q(z)$ leaves $\mathscr{R}$ through its bottom side and $\alpha<\beta$.

The following lemmas are proved exactly as Lemma 3D.1. For these lemmas we assume that $p$ and $q$ are positive constants, and $P(z)$ and $Q(z)$ are the trajectories through the points $(0, p)$ and $(0, q)$, respectively. We further assume that $P(z)$ and $Q(z)$ leave $\mathscr{R}$ with $U$-coordinate equal to $\alpha$ and $\beta$, respectively.

Lemma 3D.5. Assume that $0<p<q$ and $P(z)$ leaves $\mathscr{R}$ through its top (bottom) side. If $q-p$ is sufficiently small, then $Q(z)$ leaves $\mathscr{R}$ through its top (bottom) side and $\alpha>\beta(\alpha<\beta)$.

Lemma 3D.6. Assume that $0>p>q$ and $P(z)$ leaves $\mathscr{R}$ through its top (bottom) side. If $|q-p|$ is sufficiently small, then $Q(z)$ leaves $\mathscr{R}$ through its top (bottom) side and $\alpha>\beta(\alpha<\beta)$.

(E) How the stable and unstable trajectories vary with $\theta$. Assume, for now, that $A(z, \theta)$ is equal to either $A_{N E}(z, \theta)$ or $A_{S W}(z, \theta)$, and $B(z, \theta)$ is equal to either $B_{S E}(z, \theta)$ or $B_{N W}(z, \theta)$. For some constant $\beta$, let $l$ be the vertical line given by $U \equiv \beta$. Suppose that $A\left(z, \theta_{0}\right)$ intersects $l$ for $z=z_{0}$. Let $\gamma_{A}\left(\theta_{0}\right)=A_{2}\left(z_{0}, \theta_{0}\right)$. We assume throughout that $\gamma_{A}\left(\theta_{0}\right) \neq 0$. Then $d A_{1}\left(z_{0}, \theta_{0}\right) / d z \neq 0$, and, therefore, $A\left(z, \theta_{0}\right)$ crosses $l$ transversely. Hence, there exists a constant $\delta>0$ such that if $\left|\theta-\theta_{0}\right|<\delta$, then $A(z, \theta)$ also crosses $l$ for some $z$, say $z(\theta)$. For $\left|\theta-\theta_{0}\right|<\delta$, let $\gamma_{A}(\theta)=$ $A_{2}(z(\theta), \theta)$. There is no problem in choosing $\gamma_{A}(\theta)$ and $z(\theta)$ to be continuous.

Lemma 3E.1. Assume that $0<\theta_{1}-\theta_{0}<\delta$. Then either $0<\gamma_{A}\left(\theta_{0}\right)<\gamma_{A}\left(\theta_{1}\right)$ or $0>\gamma_{A}\left(\theta_{0}\right)>\gamma_{A}\left(\theta_{1}\right)$.

Proof. We only consider the case $A\left(z, \theta_{0}\right)=A_{N E}\left(z, \theta_{0}\right)$ since the case $A\left(z, \theta_{0}\right)=$ $A_{S W}\left(z, \theta_{0}\right)$ is similar. We shall also only treat the case $\gamma_{A}\left(\theta_{0}\right)>0$, since the case $\gamma_{A}\left(\theta_{0}\right)<0$ is also similar. We first prove the result if $|\beta-A|$ is small. Suppose that $F^{\prime \prime}(A)=-\alpha$. Of course, $\alpha>0$. Let

$$
M(\theta)=\left[\begin{array}{ll}
0 & 1 \\
\alpha & \theta
\end{array}\right] \text { and } W=\left[\begin{array}{l}
U \\
V
\end{array}\right]
$$

If we linearize (1.3) at $\bar{A}$ we obtain the system $W^{\prime}=M(\theta) W$. The eigenvalues of $M(\theta)$ are

$$
\lambda_{1}(\theta)=\left(\theta+\sqrt{\theta^{2}+4 \alpha}\right) / 2 \text { and } \lambda_{2}(\theta)=\left(\theta-\sqrt{\theta^{2}+4 \alpha}\right) / 2 .
$$

An eigenvector corresponding to $\lambda_{1}(\theta)$ is $\left(1, \lambda_{1}(\theta)\right)$. Hence, $A(z, \theta)$ approaches $\bar{A}$ as $z \rightarrow-\infty$, tangent to a line through $\bar{A}$ with slope $\lambda_{1}(\theta)$. Since $\lambda_{1}^{\prime}(\theta)>0$ it follows 
that there exists a constant $\varepsilon>0$ such that the following is true. Let $\rho$ be the ray $\{(U, V): U=A+\varepsilon, V>0\}$. Clearly if $\varepsilon$ is sufficiently small, then $A\left(z, \theta_{0}\right)$ and $A\left(z, \theta_{1}\right)$ will intersect $\rho$. Choose $\xi_{0}$ and $\xi_{1}$ so that $A_{1}\left(\xi_{0}, \theta_{0}\right)=A_{1}\left(\xi_{1}, \theta_{1}\right)=A+\varepsilon$, but $A_{1}\left(\xi, \theta_{0}\right)<A+\varepsilon$ for $\xi<\xi_{0}$ and $A_{1}\left(\xi, \theta_{1}\right)<A+\varepsilon$ for $\xi<\xi_{1}$. Let $P_{0}=$ $A_{2}\left(\xi_{0}, \theta_{0}\right)$ and $P_{1}=A_{2}\left(\xi_{1}, \theta_{1}\right)$. Then $P_{0}<P_{1}$. That is, near $\bar{A}, A\left(z, \theta_{1}\right)$ lies above $A\left(z, \theta_{0}\right)$.

By following the curves $A\left(z, \theta_{0}\right)$ and $A\left(z, \theta_{1}\right)$ around in the phase plane and using an analysis similar to that given in the preceeding section, we find that the lemma is true if the curves $A\left(z, \theta_{0}\right)$ and $A\left(z, \theta_{1}\right)$ never intersect. Suppose that they did, say, at $q=\left(q_{1}, q_{2}\right)$. Choose $\eta_{0}$ and $\eta_{1}$ so that $A\left(\eta_{0}, \theta_{0}\right)=A\left(\eta_{1}, \theta_{1}\right)=q$, and $A\left(\eta_{2}, \theta_{0}\right) \neq$ $A\left(\eta_{3}, \theta_{0}\right)$ for any $\eta_{2}<\eta_{0}$ or $\eta_{3}<\eta_{1}$. We assume that $q_{2}>0$. The other cases are similar. Let

$$
\zeta_{0}=\sup \left\{z<\eta_{0}: A_{2}\left(z, \theta_{0}\right)=0\right\} \text { and } \zeta_{1}=\sup \left\{z<\eta_{1}: A_{2}\left(z, \theta_{1}\right)=0\right\} .
$$

It may be true that $\xi_{0}=\xi_{1}=-\infty$. Let $C_{0}=A_{1}\left(\zeta_{0}, \theta_{0}\right)$ and $C_{1}=A_{1}\left(\zeta_{1}, \theta_{1}\right)$. Assume for now that $\zeta_{0} \neq-\infty$. (This implies that $\zeta_{1} \neq-\infty$.) Then, it must be true that $C_{1}<C_{0}$. Hence, the curve $A\left(z, \theta_{0}\right)$ for $z \in\left(\zeta_{0}, \eta_{0}\right)$ lies below the curve $A\left(z, \theta_{1}\right)$ for $z \in\left(\zeta_{1}, \eta_{1}\right)$. Note that the same is true if $\zeta_{0}=\zeta_{1}=-\infty$. At $q, A\left(z, \theta_{0}\right)$ is tangent to the vector $W^{0} \equiv\left(q_{2}, \theta_{0} q_{2}-F\left(q_{1}\right)\right)$, while $A\left(z, \theta_{1}\right)$ is tangent to the vector $W^{1} \equiv$ $\left(q_{2}, \theta_{1} q_{2}-F\left(q_{1}\right)\right)$. Let $W^{0}=\left(W_{1}^{0}, W_{2}^{0}\right)$ and $W^{1}=\left(W_{1}^{1}, W_{2}^{1}\right)$. Since $W_{1}^{0}=W_{1}^{1}$ and $W_{2}^{0}<W_{2}^{1}$ we have the desired contradiction, and the proof of the lemma is complete.

Now let us consider $B\left(z, \theta_{0}\right)$. Suppose that $B\left(z, \theta_{0}\right)$ intersects $l$ when $z=z_{0}$. Let $\gamma_{B}\left(\theta_{0}\right)=B_{2}\left(z_{0}, \theta_{0}\right)$. If $\gamma_{B}\left(\theta_{0}\right) \neq 0$, then $B\left(z, \theta_{0}\right)$ crosses $l$ tranversely. Hence, there exists $\delta>0$ such that if $\left|\theta-\theta_{0}\right|<\delta$, then $B(z, \theta)$ also crosses $l$ for some $z$, say $z(\theta)$. Let $\gamma(\theta)=B_{2}(z(\theta), \theta)$. A proof similar to the one given for Lemma 3E.1 proves the following lemma.

Lemma 3E.2. Assume that $0<\theta_{1}-\theta_{0}<\delta$. Then either $0<\gamma_{B}\left(\theta_{1}\right)<\gamma_{B}\left(\theta_{0}\right)$ or $0>\gamma_{B}\left(\theta_{1}\right)>\gamma_{B}\left(\theta_{0}\right)$.

4. Proof of the propositions. We assume throughout this section that the array at $\theta=\theta_{0}$ is given by

$$
\mathscr{A}\left(\theta_{0}\right)=\frac{T_{1} T_{2} \cdots T_{n}}{B_{1} B_{2} \cdots B_{m}}
$$

(A) Proof of Proposition 2.2. Assume that there exists an $A \rightarrow B$ connection with speed $\theta_{1}$ where $\theta_{1}<\theta_{0}$, and that there do not exist any connections with speed $\theta \in\left(\theta_{1}, \theta_{0}\right)$. We wish to prove that there exists an integer $k$ such that either $A^{*}=T_{k}$ and $B^{*}=T_{k+1}$, or $A^{*}=B_{k+1}$ and $B^{*}=B_{k}$. We only consider the case when $A<B$ and $A_{N E}\left(z, \theta_{1}\right)=B_{N W}\left(z, \theta_{1}\right)$ since the other cases are similar. To simplify the notation we set $A(z, \theta)=A_{N E}(z, \theta)$ and $B(z, \theta)=B_{N W}(z, \theta)$. Furthermore, we let

$$
A(z, \theta)=\left(A_{1}(z, \theta), A_{2}(z, \theta)\right) \text { and } B(z, \theta)=\left(B_{1}(z, \theta), B_{2}(z, \theta)\right) \text {. }
$$

In what follows the reader should refer to Figure 8(A). 
Choose $P$ so that $P<B$ and $F$ does not have any critical points in the interval $(P, B)$. Let $l$ be the line $U \equiv P$. Clearly, $B\left(z, \theta_{1}\right)$ must intersect $l$ at least once. Let

$$
z_{0}=\sup \left\{z \mid B_{1}\left(z, \theta_{1}\right)=P\right\} \quad \text { and } \gamma=B_{2}\left(z_{0}, \theta_{1}\right) .
$$

Note that $\gamma>0$. For $\theta$ sufficiently close to $\theta_{1}$, there exist continuous functions $\gamma_{A}(\theta)$ and $\gamma_{B}(\theta)$ such that $\gamma_{A}\left(\theta_{1}\right)=\gamma_{B}\left(\theta_{1}\right)=\gamma, A(z, \theta)$ intersects $l$ at a point whose $V$ coordinate is $\gamma_{A}(\theta)$, and $B(z, \theta)$ intersects $l$ at a point whose $V$ coordinate is $\gamma_{B}(\theta)$. We assume that $\gamma_{A}(\theta)$ and $\gamma_{B}(\theta)$ are defined for $\theta \in\left(\theta_{1}-\varepsilon, \theta_{1}+\varepsilon\right)$. We also assume that $\varepsilon<\delta$ where $\delta$ appears in Lemmas 3E.1 and 3E.2.

Choose $\theta_{2}$ so that $0<\theta_{2}-\theta_{1}<\varepsilon$ and $\theta_{2}<\theta_{0}$. We conclude from Lemmas 3E.1 and $3 \mathrm{E} .2$ that $\gamma_{A}\left(\theta_{2}\right)>\gamma_{B}\left(\theta_{2}\right)$. That is, as they cross $l, A\left(z, \theta_{2}\right)$ lies above $B\left(z, \theta_{2}\right)$. Since $A\left(z, \theta_{2}\right)$ cannot intersect $B\left(z, \theta_{2}\right)$, this implies that $A\left(z, \theta_{2}\right)$ crosses the axis $U=B$ above $\bar{B}$. More precisely, there exists a constant $\zeta_{0}$ such that $A_{1}\left(\zeta_{0}, \theta_{2}\right)=B$ and $A_{2}\left(\zeta_{0}, \theta_{2}\right)>0$. Furthermore, setting $\lambda=A_{2}\left(\zeta_{0}, \theta_{2}\right)$, we can make $\lambda$ as small as we please by choosing $\theta_{2}-\theta_{1}$ small. In particular, by choosing $\theta_{2}-\theta_{1}$ sufficiently small we may conclude that $A\left(z, \theta_{2}\right)$ and $B_{N E}\left(z, \theta_{2}\right)$ exit the same side of the rectangle $\mathscr{R}$, at points which can be made arbitrarily close to each other. From the discussion in subsection 3(D) it follows that if $B_{N E}\left(z, \theta_{2}\right)$ exits the top side of $\mathscr{R}$, then $A\left(z, \theta_{2}\right)$ exits $\mathscr{R}$ immediately to the left of $B_{N E}\left(z, \theta_{2}\right)$. If, on the other hand, $B_{N E}\left(z, \theta_{2}\right)$ exits the bottom side of $\mathscr{R}$, then $A\left(z, \theta_{2}\right)$ exits $\mathscr{R}$ directly to the right of $B_{N E}\left(z, \theta_{2}\right)$. Putting these facts together we have proven that if the array at $\theta_{2}$ is

$$
\mathscr{A}\left(\theta_{2}\right)=\frac{p_{1} p_{2} \cdots p_{\alpha}}{q_{1} q_{2} \cdots q_{\beta}}
$$

then there exists an integer $k$ such that either $A^{*}=p_{k}$ and $B^{*}=p_{k+1}$, or $A^{*}=q_{k+1}$ and $B^{*}=q_{k}$.

To complete the proof we show that $\mathscr{A}\left(\theta_{0}\right)=\mathscr{A}\left(\theta_{2}\right)$. This follows from the assumption that there do not exist any connections with speed $\theta \in\left(\theta_{2}, \theta_{0}\right)$. Since there are no connections, all of the unstable trajectories vary continuously with $\theta$. In particular, the array remains the same for $\theta \in\left(\theta_{2}, \theta_{0}\right)$.

(B) Proof of Proposition 2.3. Suppose that $\theta_{1}<\theta_{0}$, there exists an $A \rightarrow B$ connection with speed $\theta_{1}$, and there do not exist any other connections with speed
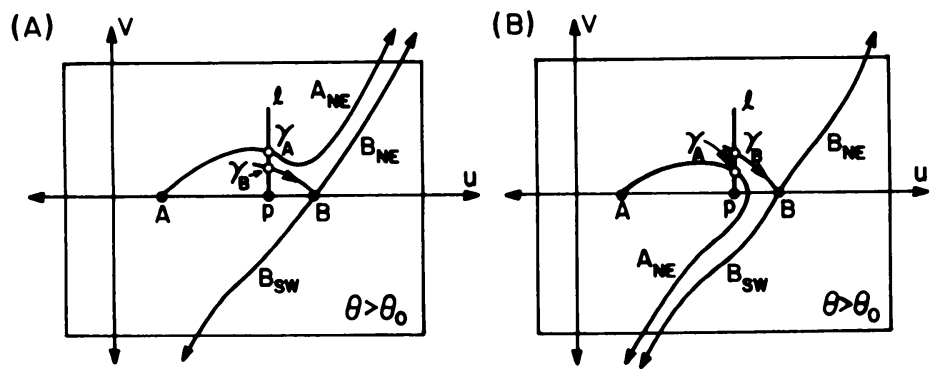

Figure 8. (A) For $\theta$ a little bigger than $\theta_{0}, A_{N E}(\theta, z)$ crosses $l$ just above $B_{N W}(z, \theta)$. This forces $A_{\mathrm{N}:}(z, \theta)$ to leave $R$ just to the left of $B_{N I:}(z, \theta)$.

(B) For $\theta$ a little smaller than $\theta_{0}, A_{N l}(\theta, z)$ crosses $l$ just below $B_{N W}(z, \theta)$. This forces $A_{N E}(z, \theta)$ to leave $R$ just to the left of $B_{N E}(z, \theta)$. 
$\theta \in\left(\theta_{1}, \theta_{0}\right)$. From Proposition 2.2, it follows that if the array at $\theta=\theta_{0}$ is given by (4.1), then there exists an integer $k$ such that either $A^{*}=T_{k}$ and $B^{*}=T_{k+1}$, or $A^{*}=B_{k+1}$ and $B^{*}=B_{k}$. We assume that $A^{*}=T_{k}$ and $B^{*}=T_{k+1}$ since the proof of the other case is quite similar. We also assume that $A<B$ and $A_{N E}\left(z, \theta_{1}\right)=$ $B_{N W}\left(z, \theta_{1}\right)$, since, again, the proof of the other cases are similar. As before we set $A(z, \theta)=A_{N E}(z, \theta)$ and $B(z, \theta)=B_{N W}(z, \theta)$. The proof now proceeds in a fashion quite similar to the proof of Proposition 2.2. In what follows the reader should refer to Figure 8(B).

Let $P, l, \varepsilon, \gamma_{A}(\theta)$ and $\gamma_{B}(\theta)$ be as in the proof of Proposition 2.2. Choose $\theta_{2}$ so that $0<\theta_{1}-\theta_{2}<\varepsilon$. Lemmas 3E.1 and 3E.2 now imply that $\gamma_{A}\left(\theta_{2}\right)<\gamma_{B}\left(\theta_{2}\right)$. This implies that $A\left(z, \theta_{2}\right)$ must cross the $U$-axis immediately to the left of $\bar{B}$. More precisely, there exists $\zeta_{0}$ such that $A_{2}\left(\zeta_{0}, \theta_{2}\right)=0$ and $A_{1}\left(\zeta_{0}, \theta_{2}\right)<B$. Furthermore, if $\lambda=A_{1}\left(\zeta_{0}, \theta_{2}\right)$, then $B-\lambda$ can be made as small as we please by choosing $\theta_{1}-\theta_{2}$ small. In particular, by choosing $\theta_{1}-\theta_{2}$ sufficiently small we may conclude that $A\left(z, \theta_{2}\right)$ and $B_{s W}\left(z, \theta_{2}\right)$ exit the same side of the rectangle $\mathscr{R}$, at points which can be made arbitrarily close to each other. From the discussion in subsection 3(D) it follows that if $B_{S W}\left(z, \theta_{0}\right)$ exits the top side of $\mathscr{R}$, then $A\left(z, \theta_{0}\right)$ exits $\mathscr{R}$ immediately to the right of $B_{S W}\left(z, \theta_{2}\right)$. If $B_{S W}\left(z, \theta_{2}\right)$ exits the bottom side of $\mathscr{R}$, then $A\left(z, \theta_{2}\right)$ exits $\mathscr{R}$ immediately to the left of $B_{S W}\left(z, \theta_{2}\right)$. Since $B_{S W}(z, \theta)$ corresponds to the 'other $B$ ' in the statement of Proposition 2.3, this completes the proof.

We now discuss what happens if two or more waves exit with the same speed. Here we only consider the case when there exists two waves with the same speed. The case for more than two waves is quite similar. So suppose that there exist $A \rightarrow B$ and $C \rightarrow D$ connections with speed $\theta_{0}$. If $B \neq C$, then there is no problem in applying Proposition 2.3 to determine how the array changes after the connections. The $A$ goes next to the other $B$, while $C$ goes next to the other $D$. An example is

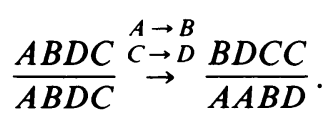

If $B=C$, then there are two cases to consider, depending on if $B$ and $C$ correspond to the same entry in the array. Recall that each integer appears twice in an array. If $B$ and $C$ are the same entry, then we must consider the $A \rightarrow B$ connection first and then the $C \rightarrow D$ connection. That is, we first put the $A$ next to the other $B$, and then put the $C$, which is now $B$, next to the other $D$. An example is

$$
\frac{A B D}{A B D} \stackrel{\substack{A \rightarrow B \\ B \rightarrow D}}{\rightarrow} \frac{D}{A A B B D} .
$$

If $B$ and $C$ correspond to different entries, then we first consider the $B \rightarrow D$ connection and then the $A \rightarrow B$ connection. An example is

$$
\frac{D A A B}{D B} \stackrel{\substack{A \rightarrow B \\ B \rightarrow D}}{\rightarrow} \frac{D B A A B}{D} .
$$

The proofs of these results are quite similar to the proof of Proposition 2.3. 
(C) Proof of Proposition 2.4. We show that given $A, B$, and $\theta_{0}>0$, there can exist at most a finite number of $A \rightarrow B$ connections with speeds greater than $\theta_{0}$. Since we are assuming that there are only a finite number of critical points this certainly implies the desired result.

In subsection $3(\mathrm{~B})$ it was shown that there exists $\theta_{1}$ such that no $A \rightarrow B$ connection exists for $\theta>\theta_{1}$. Certainly if no $A \rightarrow B$ connection exists with speed $\theta_{2}$, then there exists $\varepsilon>0$ such that no $A \rightarrow B$ connections exist with speed $\theta \in\left(\theta_{2}-\right.$ $\left.\varepsilon, \theta_{2}+\varepsilon\right)$. To complete the proof we note that Propositions 2.2 and 2.3 imply that if there exists an $A \rightarrow B$ connection with speed $\theta_{3}$ then there exists $\varepsilon>0$ such that no other $A \rightarrow B$ connection exists with speed $\theta \in\left(\theta_{3}-\varepsilon, \theta_{3}+\varepsilon\right)$.

(D) Proof of Proposition 2.1. Suppose that $A\left(\theta_{0}\right)$ is given by (4.1) and $T_{k}<T_{k+1}$. We show that there exists a $T_{k} \rightarrow T_{k+1}$ connection for some $\theta<\theta_{0}$. We do not discuss the case when $B_{k+1}<B_{k}$ since the proof is similar. Assume that $A$ and $B$ are the critical points which satisfy $A^{*}=T_{k}$ and $B^{*}=T_{k+1}$. Now $T_{k}$ and $T_{k+1}$ correspond to two points on the top side of $\mathscr{R}$ which lie on an unstable trajectory of $\bar{A}$ and $\bar{B}$, respectively. Let these points be $P_{A}$ and $P_{B}$. We denote the unstable trajectory on which $P_{A}$ lies by $A(z)$ and the unstable trajectory on which $P_{B}$ lies by $B(z)$. There are now a number of cases to consider. These are

$\begin{array}{llll}\text { (4D.1a) } & A<B, & A(z)=A_{N E}\left(z, \theta_{0}\right), & B(z)=B_{N E}\left(z, \theta_{0}\right), \\ \text { (4D.1b) } & A<B, & A(z)=A_{S W}\left(z, \theta_{0}\right), & B(z)=B_{N E}\left(z, \theta_{0}\right), \\ \text { (4D.1c) } & A<B, & A(z)=A_{N E}\left(z, \theta_{0}\right), & B(z)=B_{S W}\left(z, \theta_{0}\right), \\ \text { (4D.1d) } & A<B, & A(z)=A_{S W}\left(z, \theta_{0}\right), & B(z)=B_{S W}\left(z, \theta_{0}\right), \\ \text { (4D.1e) } & A>B, & A(z)=A_{N E}\left(z, \theta_{0}\right), & B(z)=B_{N E}\left(z, \theta_{0}\right), \\ \text { (4D.1f) } & A>B, & A(z)=A_{S W}\left(z, \theta_{0}\right), & B(z)=B_{N E}\left(z, \theta_{0}\right), \\ \text { (4D.1g) } & A>B, & A(z)=A_{N E}\left(z, \theta_{0}\right), & B(z)=B_{S W}\left(z, \theta_{0}\right), \\ \text { (4D.1h) } & A>B, & A(z)=A_{S W}\left(z, \theta_{0}\right), & B(z)=B_{S W}\left(z, \theta_{0}\right) .\end{array}$

We first consider (4D.1a). We prove that $A_{N E}(z, \theta)=B_{N W}(z, \theta)$ for some $\theta \in$ $\left(0, \theta_{0}\right)$. This is done using a shooting argument. To set up the shooting argument we first consider, in detail, how the trajectories $A(z)$ and $B(z)$ spiral around in the phase plane as they are followed backwards from the points $P_{A}$ and $P_{B}$.

Choose $\left\{z_{k}\right\}, k=1,2, \ldots$, so that, for each $k, z_{k}>z_{k+1}$, and $B_{2}(z)=0$ if and only if $z=z_{k}$ for some $k$. Let $\gamma_{k}=B_{1}\left(z_{k}\right)$. That is, $\gamma_{k}$ is the $U$-coordinate of the $k$ th place where the trajectory $B(z)$ intersects the $U$-axis as it is followed backwards from $P_{B}$.

We claim that either $\gamma_{1}=B$ or $\gamma_{1}<A$. To prove that it is impossible for $\gamma_{1}>B$ we use the fact that $U^{\prime}=V$ and, therefore, all trajectories spiral in the counterclockwise direction as they are followed backwards. This fact implies that $\gamma_{k}>\gamma_{1}$, for all $k$. Therefore, if $\gamma_{1}>B$ it would be impossible for $\lim _{z \rightarrow-\infty} B(z)=\bar{B}$. We now wish to show that it is impossible for $\gamma_{1} \in(A, B)$. If this were true, then, using (1.6), which implies that $F\left(\gamma_{1}\right)>F(B)$, it follows that there exists a critical point 
$C \in(A, B)$ such that $F(C)>F(B)$. We assume that $C$ is chosen so that $F(C)>$ $F(U)$ for all $U \in(A, B)$. Now $C_{N E}\left(z, \theta_{0}\right)$ can never cross the $U$-axis between $A$ and $B$. This is because of (1.6). Suppose that $\gamma_{1} \in(C, B)$. Then $C_{N E}\left(z, \theta_{0}\right)$ must exit the top side of $\mathscr{R}$ between $A(z)$ and $B(z)$ which is a contradiction. Now suppose that $\gamma_{1} \in(A, C)$. It then follows that $B(z)$ must cross both $C_{S W}(z, 0)$ and $C_{N W}(z, 0)$. Since $H(U, V)=F(C)$ on both of these trajectories, (1.6) gives the desired contradiction. Note that this, together with Proposition 2.2, implies that if $A<B$ and there exists a critical point $C$ such that $A<C<B$ and $F(C)>\max \{F(B), F(A)\}$, then there cannot exist an $A \rightarrow B$ or $B \rightarrow A$ connection.

So it remains to consider the cases $\gamma_{1}=B$ and $\gamma_{1}<A$. We shall assume that $\gamma_{1}<A$ since this is more difficult than the case $\gamma_{1}=B$. In what follows the reader should refer to Figure 9. Note that $\gamma_{r}=B$ for some integer $r$. Following $B(z)$ backwards from $P_{B}$ and using the fact that the trajectories spiral in the counterclockwise direction as they are followed backwards, we find that $r$ is an odd number and

$$
\gamma_{1}<\gamma_{3}<\cdots<\gamma_{r-2}<A<B=\gamma_{r}<\gamma_{r-1}<\cdots<\gamma_{4}<\gamma_{2} \text {. }
$$

It is impossible for $\gamma_{k} \in(A, B)$ for some $k$ because of the same reason that $\gamma_{1} \notin(A, B)$. We also find that $B_{2}(z)>0$ for $z \in\left(z_{k+1}, z_{k}\right)$ if $k$ is even, and $B_{2}(z)<0$ for $z \in\left(z_{k+1}, z_{k}\right)$ if $k$ is odd.

We now follow $A(z)$ backwards from $P_{A}$. Its behavior is determined by noting that it must keep spiraling without ever crossing $B(z)$. Choose $\left\{z^{k}\right\}$ so that $z^{k}>z^{k+1}$ for each $k$ and $A_{2}(z)=0$ if and only if $z=z^{k}$ for some $k$. Let $\delta^{k}=A_{1}\left(z^{k}\right)$. That is, $\delta^{k}$ is the $U$ coordinate of the $k$ th place where the trajectory $A(z)$ intersects the $U$-axis as it is followed backwards from $P_{A}$. Using induction we find that if $k$ is odd, $k \leqslant r$, then $\gamma_{k-2}<\delta^{k}<\gamma_{k}$, and $A_{2}(z)<0$ for $z \in\left(z^{k+1}, z^{k}\right)$. If $k$ is even, $k<r$, then $\gamma_{k}<\delta^{k}<\gamma_{k-2}$, and $A_{2}(z)>0$ for $z \in\left(z^{k+1}, z^{k}\right)$. Furthermore, if $k$ is odd, then $\delta^{k} \leqslant A<\delta^{k-1}$. Since $r$ is odd and $\gamma_{r}=B$ we have that

$$
\begin{gathered}
\delta^{r} \leqslant A<\delta^{r-1}, \\
A_{2}(z)>0 \text { for } z \in\left(z^{r}, z^{r-1}\right) .
\end{gathered}
$$

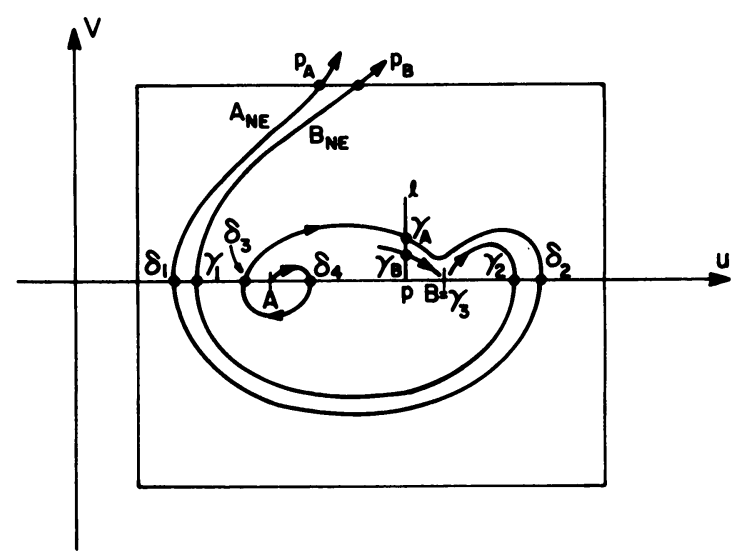

FIGURE 9 
Recall that we are trying to use a shooting argument in order to prove that $A_{N E}(z, \theta)=B_{N W}(z, \theta)$ for some $\theta \in\left(0, \theta_{0}\right)$. We are now in a position to start setting up the shooting argument. Choose $P$ so that $P<B$ and $F$ does not have a critical point in $[P, B)$. Let $l$ be the vertical line $U \equiv P$. From (4D.3a) and (4D.3b) it follows that there exists $\eta_{0} \in\left(z^{r}, z^{r-1}\right)$ such that $A_{1}\left(\eta_{0}\right)=P$. Let $\gamma_{A}\left(\theta_{0}\right)=A_{2}\left(\eta_{0}\right)$. For $\theta$ close to $\theta_{0}$ the trajectory $A_{N E}(z, \theta)$ must also intersect the line $l$. Let $\gamma_{A}(\theta)$ be the $V$ coordinate of this point of intersection. Clearly, $\gamma_{A}(\theta)$ can be chosen to be a continuous function of $\theta$, at least in some neighborhood of $\theta=\theta_{0}$. We assume that $\gamma_{A}(\theta)$ is defined on the maximum possible interval so that it is continuous.

For each $\theta>0, B_{N W}(z, \theta)$ must intersect $l$ at least once. Let $\gamma_{B}(\theta)$ be equal to the $V$ coordinate of the first place where $B_{N W}(z, \theta)$ intersects $l$ as it is followed backwards from the saddle $(B, 0)$. Note that $\gamma_{B}(\theta)$ is a continuous function for all $\theta>0$. It follows from (4D.3a) and (4D.3b) that

$$
\gamma_{A}\left(\theta_{0}\right)>\gamma_{B}\left(\theta_{0}\right) \text {. }
$$

We now demonstrate that if $\theta_{1}<\theta_{0}$ and no $A \rightarrow B$ connections exist for $\theta \in$ $\left[\theta_{1}, \theta_{0}\right]$, then $\gamma_{A}(\theta)$ is a well-defined continuous function in the interval $\left[\theta_{1}, \theta_{0}\right]$. If this were not true then there must exist $\theta_{2} \in\left(\theta_{1}, \theta_{0}\right)$ and a critical point $C$ such that an $A \rightarrow C$ connection exists with speed $\theta_{2}$. Furthermore, this connection must involve $A_{N E}\left(z, \theta_{2}\right)$. That is, either

$$
A_{N E}\left(z, \theta_{2}\right)=C_{N W}\left(z, \theta_{2}\right) \text { or } A_{N E}\left(z, \theta_{2}\right)=C_{S E}\left(z, \theta_{2}\right) .
$$

It now follows that if $\lambda=\lim _{\theta \downarrow \theta_{2}} \gamma(\theta)$, then the point $(P, \lambda)$ lies on either $C_{N E}\left(z, \theta_{2}\right)$ or $C_{S W}\left(z, \theta_{2}\right)$. Suppose that $(P, \lambda)$ lies on $C_{N E}\left(z, \theta_{2}\right)$. Let $\gamma_{c}(\theta)$ be the $V$ coordinate of the place where $C_{N E}(z, \theta)$ intersects the line $l$, chosen so that $\gamma_{c}(\theta)$ is a continuous function for $\theta$ close to $\theta_{2}$. From Lemma 3E.1 it follows that $\gamma_{c}(\theta)<\gamma_{A}(\theta)$ for $\theta$ close to but greater than $\theta_{2}$. If $\theta-\theta_{2}$ is sufficiently small, then from the way $\lambda$ was defined it follows that $\gamma_{B}(\theta)<\gamma_{c}(\theta)$.

We summarize what has been proven so far as follows. Say that $\theta$ has property star if $\theta \in\left[\theta_{1}, \theta_{0}\right]$ and there exists a critical point $C$ such that either $C_{N E}(z, \theta)$ or $C_{S W}(z, \theta)$ crosses $l$ at some point $(P, \lambda)$ which satisfies $\gamma_{B}(\theta)<\lambda<\gamma_{A}(\theta)$. Let $H=\{\theta: \theta$ has property star $\}$. We have shown that $H$ is nonempty if $\gamma_{A}(\theta)$ is discontinuous at some $\theta_{2} \in\left(\theta_{1}, \theta_{0}\right)$. We shall now show that $H$ is indeed empty.

To begin with, note that $\theta_{0} \notin H$. This is because if $\theta_{0} \in H$, then the discussion in subsection 3(D) implies that there exists a critical point $C$ such that either $C_{N E}\left(z, \theta_{0}\right)$ or $C_{S W}\left(z, \theta_{0}\right)$ leaves $\mathscr{R}$ between $A_{N E}\left(z, \theta_{0}\right)$ and $B_{N E}\left(z, \theta_{0}\right)$. This, however, contradicts the assumptions of the proposition.

Set $\theta_{3}=\sup \{\theta: \theta \in H\}$. Some sort of connection must occur with speed $\theta_{3}$. This is because the stable and unstable manifolds of the critical points vary continuously with $\theta$, except at those values of $\theta$ for which there exists a connection. Suppose, for example, that there exists an $A \rightarrow C$ connection with speed $\theta_{3}$. If, for example, $A\left(z, \theta_{3}\right)=C_{N W}\left(z, \theta_{3}\right)$, then the proof of Proposition 2.2 and the discussion in subsection 3(D) imply that there exists $\delta>0$ such that if $0<\theta-\theta_{3}<\delta$, then $C_{N E}(z, \theta)$ crosses $l$ between $\gamma_{B}(\theta)$ and $\gamma_{A}(\theta)$. That is, if $0<\theta-\theta_{3}<\delta$, then $\theta \in H$. 
This, however, contradicts the definition of $\theta_{3}$. If, on the other hand, $A\left(z, \theta_{3}\right)=$ $C_{S E}\left(z, \theta_{3}\right)$, then there exists $\delta>0$ such that $\theta \in\left(\theta_{3}, \theta_{3}+\delta\right)$ implies that $C_{S W}(z, \theta)$ crosses $l$ between $\gamma_{B}(\theta)$ and $\gamma_{A}(\theta)$. This again contradicts the definition of $\theta_{3}$.

The only other possible thing that can go wrong is that there exists a $C \rightarrow B$ connection with speed $\theta_{3}$. That is, either $C_{N E}\left(z, \theta_{3}\right)=B_{N W}\left(z, \theta_{3}\right)$ or $C_{S W}\left(z, \theta_{3}\right)=$ $B_{N W}\left(z, \theta_{3}\right)$. In either case, the proof of Proposition 2.2 and the discussion in subsection 2(D) shows that there exists $\delta>0$ such that for $\theta \in\left(\theta_{3}, \theta_{3}+\delta\right)$ the corresponding unstable trajectory from $C$ crosses $l$ between $\gamma_{B}(\theta)$ and $\gamma_{A}(\theta)$. This contradicts the definition of $\theta_{3}$ and, therefore, completes the proof that $\gamma_{A}(\theta)$ is continuous in $\left[\theta_{1}, \theta_{0}\right]$.

The proof of Proposition 2.1, case (4D.1a), will be complete if we can show that $\gamma_{A}(\theta)<\gamma_{B}(\theta)$ for some $\theta<\theta_{0}$. This, however, is true because the discussion in subsection 2(A) and the assumption that $F(A)<F(B)$ implies that $A_{N E}(z, 0)$ lies below $B_{N W}(z, 0)$.

To complete the proof of Proposition 2.1 we briefly consider the other cases, (4D.1b)-(4D.1h). If (4D.1b) is satisfied, then the proof is very similar to the one just given. One proves that, for some $\theta \in\left(0, \theta_{0}\right), A_{S W}(z, \theta)=B_{N W}(z, \theta)$. We claim that it is impossible for cases (4D.1c) and (4D.1d) to occur. We only show that (4D.1c) is impossible since the corresponding proof for (4D.1d) is similar. What are shall prove is the following. Suppose that $A<B, F(A)<F(B)$, and both the trajectories $A_{N E}\left(z, \theta_{0}\right)$ and $B_{S W}\left(z, \theta_{0}\right)$ leave the rectangle $\mathscr{R}$ on the top side. Suppose that the points where these trajectories cross the top of $\mathscr{R}$ are, respectively, $P_{A}=\left(p_{1}, V_{1}\right)$ and $Q_{B}=\left(q_{1}, V_{1}\right)$. Then, either $q_{1}<p_{1}$, or $B_{N E}\left(z, \theta_{0}\right)$ crosses the top side of $\mathscr{R}$ at a point which lies between $P_{A}$ and $Q_{B}$. That is, if $q_{1}>p_{1}$, then $A_{N E}\left(z, \theta_{0}\right)$ and $B_{S W}\left(z, \theta_{0}\right)$ cannot be "neighbors" and (4D.1c) is, therefore, impossible.

The idea of the proof is similar to the one just given. We start at $P_{A}$ and $Q_{B}$ and work our way backwards along the trajectories $A_{N E}\left(z, \theta_{0}\right)$ and $B_{S W}\left(z, \theta_{0}\right)$. As before, in order to simplify the notation, we set $A(z)=A_{N E}\left(z, \theta_{0}\right)$ and $B(z)=$ $B_{S W}\left(z, \theta_{0}\right)$. Also, let

$$
A(z)=\left(A_{1}(z), A_{2}(z)\right) \text { and } B(z)=\left(B_{1}(z), B_{2}(z)\right) \text {. }
$$

Let $\left\{z_{k}\right\}$ be chosen so that $z_{k}>z_{k+1}$ for each $k$ and $B_{2}(z)=0$ if and only if $z=z_{k}$ for some $k$. Let $\gamma_{k}=B_{1}\left(z_{k}\right)$. As before we find that either $\gamma_{1}=B$ or $\gamma_{1}<A$. We only consider the more difficult case $\gamma_{1}<A$. Choose $r$ so that $\gamma_{r}=B$. Then, as before, $r$ is even, and

$$
\gamma_{1}<\gamma_{3}<\cdots<\gamma_{r-1}<A<B=\gamma_{r}<\gamma_{r-2}<\cdots<\gamma_{4}<\gamma_{2} .
$$

Furthermore, $B_{2}(z)>0$ for $z \in\left(z_{k+1}, z_{k}\right)$ if $k$ is even, and $B_{2}(z)<0$ for $z \in$ $\left(z_{k+1}, z_{k}\right)$ if $k$ is odd.

We now follow $A(z)$ backwards from $P_{A}$. Choose $\left\{z^{k}\right\}$ so that $z^{k}>z^{k+1}$ for each $k$, and $A_{2}(z)=0$ if and only if $z=z^{k}$ for some $k$. Let $\delta^{k}=A_{1}\left(z^{k}\right)$. Using induction we find that if $k \leqslant r$ is odd, then $\gamma_{k-2}<\delta^{k}<\gamma_{k}$, and $A_{2}(z)<0$ for $z \in\left(z^{k+1}, z^{k}\right)$. If $k \leqslant r$ is even, then $\gamma_{k}<\delta^{k}<\gamma_{k-2}$, and $A_{2}(z)>0$ for $z \in\left(z^{k+1}, z^{k}\right)$. Since $r$ is even and $\gamma_{r}=B$, we find that $B<\delta^{r}$, and for $z \in\left(z^{r+1}, z^{r}\right), A_{2}(z)>0$.

We now wish to investigate what happens to $B_{N E}\left(z, \theta_{0}\right)$. To simplify the notation we set $\beta(z)=B_{N E}\left(z, \theta_{0}\right)$, with $\beta(z)=\left(\beta_{1}(z), \beta_{2}(z)\right)$. We claim that there exists a 
number $\xi_{0}$ such that $\beta_{2}\left(\xi_{0}\right)=0, \beta_{2}(z)>0$ for $z \in\left(-\infty, \xi_{0}\right)$, and $B<\beta_{1}\left(\xi_{0}\right)<\delta^{r}$. This is because $\beta(z)$ leaves the saddle $(B, 0)$ into the quarter plane $U>B, V>0$. As long as $V>0$, we have that $U^{\prime}>0$ and $B(z)$ continues to move to the right. This cannot happen forever because $B(z)$ would have to eventually intersect the trajectory $A(z)$. Hence, $B(z)$ must cross the $U$-axis at some value of $z$, say $z=\xi_{0}$. Clearly $B<B_{1}\left(\xi_{0}\right)<\delta^{r}$. Using the results of the discussion in subsection 3(D) we now conclude that $B(z)$ leaves $\mathscr{R}$ between $A(z)$ and $B(z)$. This is what we wished to show.

To complete the proof of Proposition 2.1 we must consider cases (4D.1e)-(4D.1h). Since these cases are treated in a manner very similar to the above proofs, we only point out that cases (4D.1e) and (4D.1f) are impossible. In case (4D.1g) there exists a $\theta \in\left(0, \theta_{0}\right)$ at which speed $A_{N E}(z, \theta)=B_{S E}(z, \theta)$. In case (4D.1h) there exists a $\theta \in\left(0, \theta_{0}\right)$ at which speed $A_{S W}(z, \theta)=B_{S E}(z, \theta)$.

\section{Labelled directed graph homomorphisms.}

(A) Introduction. Figure 10 illustrates the directed graph for the function $F$ shown in Figure 1. We see that even when $F$ has only four local maxima, the directed graph can be quite complicated. If $F$ were to have many more critical points, then the corresponding directed graph would be so complicated that it would be impossible

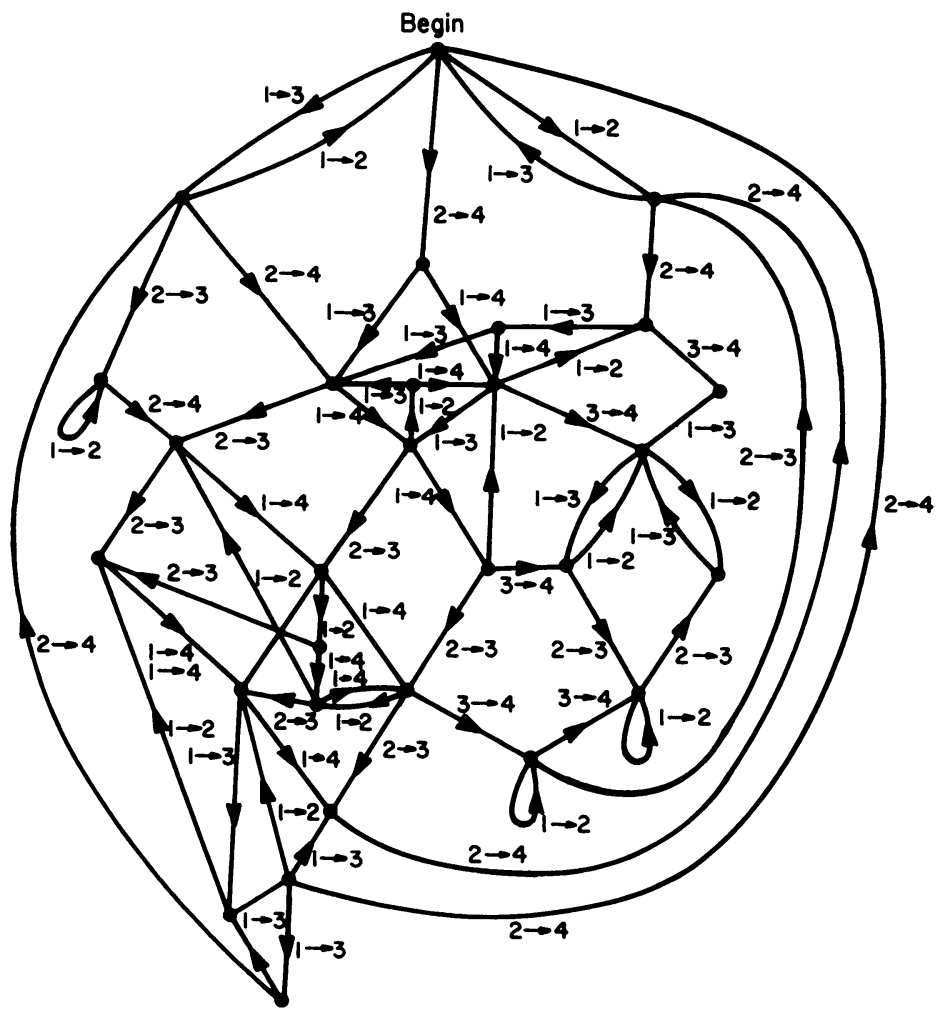

Figuri: 10. The complete directed graph associated with the equivalence of class functions shown in Figure 1. 
to analyze directly. We shall show that if $A$ and $B$ are distinct critical points of $F$ and if we are only interested in determining the number of $A \rightarrow B$ connections, then it is not usually necessary to consider the entire directed graph corresponding to $F$. The reason is that many of the critical points of $F$ play very little role as far as the $A \rightarrow B$ connections are concerned. For example, suppose that $F$ is as shown in Figure 11.

Then, as we shall see later, the only critical points which play a crucial role, as far as the $A \rightarrow B$ connections are concerned, are $A, B, C$ and $D$. Let $G_{F}$ be the directed graph corresponding to $F$. Since the $A \rightarrow B$ connections really only depend on four critical points, there should exist a much simpler graph, which we denote by $\Gamma(A, B)$, which contains all the essential information of $G_{F}$ as far as the $A \rightarrow B$ connections are concerned. We prove that there does indeed exist such a graph $\Gamma(A, B)$. In fact, what we prove is that there exists a mapping $\Phi$ from $G_{F}$ onto $\Gamma(A, B)$ which preserves enough of the structure of $G_{F}$ so that if we are interested in determining the number of $A \rightarrow B$ connections, then it is only necessary to consider the graph $\Gamma(A, B)$.

The next few sections are devoted to making these statements precise. We begin by defining what is meant by a labelled graph homomorphism. We then describe how to choose the graphs $\Gamma(A, B)$. After stating and proving the main theorem, we prove some general results about how many connections exist between two given stable critical points. In particular, we prove that if $F, A$ and $B$ are as shown in Figure 8, then there exists an infinite number of $A \rightarrow B$ connections.

(B) Labelled directed graphs, paths and mappings between graphs. We begin by giving a precise definition of what we mean by a labelled directed graph, or LDG for short. An LDG consists of four objects. If $G$ is an $\mathrm{LDG}$ we write $G=(X, E, Y, \Theta)$. Here, $X$ is a finite set, say $X=\left\{X_{1}, \ldots, X_{K}\right\} . X$ denotes the set of nodes. Each node in the graph corresponds, in our situation, to an array of integers. The set of edges is a subset of $X \times X$, which we denote by $\hat{E}$. Thus $\left(X_{j}, X_{k}\right)$ denotes the edge which begins at $X_{j}$ and ends at $X_{k}$. It is convenient to write $X_{k}=e * X_{j}$. Let $\hat{E}\left(X_{k}\right)$ equal the set of edges whose first component is $X_{k}$. We then set $E\left(X_{k}\right)=\hat{E}\left(X_{k}\right) \cup\left\{X_{k}\right\}$ and $E=\cup E\left(X_{k}\right)$, where the union is over all nodes $X_{k}$. By definition, we write $X_{k} * X_{k}=X_{k} . Y$ is a set of nonnegative integers. It corresponds to the set of labels. Usually $Y$ is of the form $\{0,1,2, \ldots, N\}$. We denote this latter set by $Y_{N}$. Finally, $\Theta$ is a function from $E$ into $Y \times Y$. For example, if the edge $e$ corresponds to an

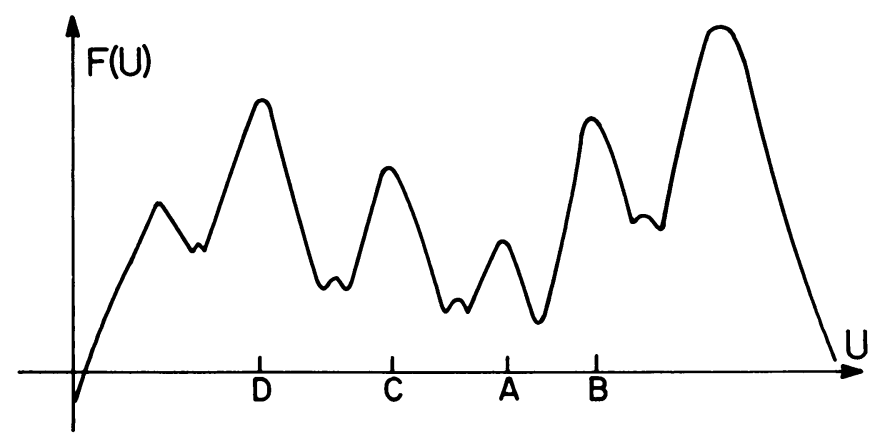

Figure 11 
$A \rightarrow B$ connection, then $\Theta(e)=\left(A^{*}, B^{*}\right)$. We always assume that $\Theta\left(X_{k}\right)=(0,0)$ for each node $X_{k}$, and if $e \in \hat{E}$ and $\Theta(e)=\left(A^{*}, B^{*}\right)$, then $A^{*} \neq 0$ and $B^{*} \neq 0$.

Let $P=\left\{e_{k}\right\}$ be a finite or infinite set of edges.

We say that $P$ is a path in $G$ if for each $k, e_{k} \in E$ and $e_{k} \in E\left(X_{k}\right)$ implies that $e_{k+1} \in E\left(e_{k} * X_{k}\right)$. If $P$ is finite, say $P=\left\{e_{1}, \ldots, e_{n}\right\}$, with $e_{1} \in E\left(X_{1}\right), e_{n} \in E\left(X_{n}\right)$ and $X_{n+1}=e_{n} * X_{n}$, then we say that $P$ is a path from $X_{1}$ to $X_{n+1}$. In this case we write $X_{n+1}=P * X_{n}$. The path $P=\left\{e_{k}\right\}$ is called admissible if the following is satisfied. For any $k$, suppose that $e_{k} \in E\left(X_{k}\right)$, and $e$ is any element of $E\left(X_{k}\right)$. We do not necessarily assume that $e=e_{k}$. Suppose that $\Theta(e)=\left(A^{*}, B^{*}\right)$. Then, there exists an integer $j$ such that $j>k$ and $\Theta\left(e_{j}\right)=\left(A^{*}, B^{*}\right)$.

Note that $F(U)$ determines a path in $G_{F}$. We denote the path by $P_{F}$. Proposition 2.1 implies that $P_{F}$ is an admissible path in $G_{F}$. Finally, we say that a node $X$ is a generator of the graph $G$ if for every node $X^{\prime}$ there exists a path from $X$ to $X^{\prime}$.

Before defining what is meant by an LDG homomorphism we must introduce some more notation. Suppose that $Y_{A}$ and $Y_{B}$ are two subsets of the nonnegative integers. We assume that $\{0\}$ is a subset of both sets. Let $g: Y_{A} \rightarrow Y_{B}$. Assume that $g(0)=0$. Then the new map $\hat{g}: Y_{A} \times Y_{A} \rightarrow Y_{B} \times Y_{B}$ is defined by

$$
\hat{g}(j, k)= \begin{cases}(0,0) & \text { if } g(j)=0 \text { or } g(k)=0, \\ (g(j), g(k)) & \text { otherwise. }\end{cases}
$$

DEFINITION 5B.1. Suppose that

$$
G_{A}=\left\{X_{A}, E_{A}, Y_{A}, \Theta_{A}\right\} \text { and } G_{B}=\left\{X_{B}, E_{B}, Y_{B}, \Theta_{B}\right\}
$$

are labelled directed graphs. Then $\Phi=\left(\Phi_{X}, \Phi_{E}, \Phi_{\theta}\right)$ is an LDG homomorphism from $G_{A}$ into $G_{B}$ if $\Phi_{X}: X_{A} \rightarrow X_{B}, \Phi_{E}: E_{A} \rightarrow E_{B}$ and $\Phi_{\theta}: Y_{A} \rightarrow Y_{B}$. Furthermore, for each $x \in X_{A}$ and $e \in E(x)$ :

(A) $\Phi_{E}(E(x)) \subset E\left(\Phi_{X}(x)\right)$,

(B) $\Phi_{X}(e * x)=\Phi_{E}(e) * \Phi_{X}(x)$,

(C) $\Phi_{E}(x)=\Phi_{X}(x)$,

(D) $\Phi_{\theta}(0)=0$, and

(E) $\hat{\Phi}_{\theta}\left(\Theta_{A}(e)\right)=\Theta_{B}\left(\Phi_{E}(e)\right)$.

In what follows we always use the same letter $\Phi$ to denote $\Phi_{X}, \Phi_{E}$ and $\Phi_{\theta}$.

Definition 5B.2. $\Phi$ is an LDG homomorphism from $G_{A}$ onto $G_{B}$ if in addition to the conditions of the preceding definition the following are satisfied:

(A) for each node $y \in X_{B}$ there exists a node $x \in X_{A}$ such that $\Phi(x)=y$;

(B) if $\Phi(x)=y$ and $f \in E(y)$, then there exists a path $\left\{e_{1}, \ldots, e_{k}\right\} \in G_{A}$ such that:

(i) $e_{1} \in E(x)$,

(ii) if $x_{0}=x$ and $x_{j}=e_{j} * x_{j-1}$ for $j=1, \ldots, k$, then $\Phi\left(x_{j}\right)=y$ and $\Phi\left(e_{j}\right)=y$ for $j=1, \ldots, k-1$, and

(iii) $\Phi\left(e_{k}\right)=f$.

The following basic facts about LDG homomorphisms follow immediately from the definitions.

LEMMA 5B.1. If $P$ is a path in $G_{A}$, and $\Phi$ is an $L D G$ homomorphism from $G_{A}$ into $G_{B}$, then $\Phi(P)$ is a path in $G_{B}$. 
LEMMA 5B.2. Suppose that $\Phi$ is an $L D G$ homomorphism from $G_{A}$ into $G_{B}$, and $\Phi$ satisfies Definition 5B.2(B). Furthermore, assume that $\Phi$ maps a generator of $G_{A}$ onto a generator of $G_{B}$. Then $\Phi$ satisfies Definition 5B.2(A).

(C) The graph $\Gamma(A, B)$. Unless stated otherwise, we assume that $A$ and $B$ are fixed critical points of $F$ with $A<B$ and $F(A)<F(B)$. We also assume that there does not exist a critical point $C$ which satisfies $A<C<B$ and $F(C)>F(B)$. In Proposition 2.3 it was shown that if there did exist such a $C$, then there could not exist any $A \rightarrow B$ connections.

We now introduce some notation which will be used throughout the rest of the paper. Let $K$ be equal to the set of critical poirits of $F$. Recall that by a critical point we mean a value of $U$, where $F$ assumes a local maximum. Furthermore, to each $C \in K$ there corresponds an integer, denoted by $C^{*}$, which is determined by the ordering of the values of $F$ at the critical points. Let $K(A)$ equal the set of critical points $C$ which satisfy $F(C) \geqslant F(A)$, and there does not exist a critical point $D$ which lies between $A$ and $C$ such that $F(D)>F(C)$. Note that $\{A, B\} \subset K(A)$. Let $\mathscr{C}(A)=\left\{C^{*}: C \in K(A)\right\}$. Let $K(A, B)=\{C: C \in K(A)$ and $F(C) \leqslant F(B)\}$ and $\mathscr{C}(A, B)=\left\{C^{*}: C \in K(A, B)\right\}$. Note that $\{A, B\} \subset K(A, B)$. Let

$$
\begin{gathered}
\mathscr{X}=K(A) \backslash K(A, B), \quad \mathscr{Y}=K \backslash K(A), \\
\mathscr{C}(\mathscr{X})=\left\{C^{*}: C \in \mathscr{X}\right\} \quad \text { and } \mathscr{C}(\mathscr{Y})=\left\{C^{*}: C \in \mathscr{Y}\right\} .
\end{gathered}
$$

The proof of Proposition 2.3 shows that if $D \in \mathscr{Y}$, then there cannot exist any $A \rightarrow D$ connections.

Let $F(A, B)(U)$ be any function which satisfies (1.2) and whose set of critical points is equal to $K(A, B)$. We also assume that if $C \in K(A, B)$, then $F(A, B)(C)$ $=F(C)$. Finally, for each positive integer $n$, let $G_{n}$ be the graph generated by the array which has the sequence of integers $\{n, n-1, \ldots, 2,1,1,2, \ldots, n-1, n\}$ on top. We assume that the generator of $G_{n}$ has no entry on its bottom. This implies that each array in $G_{n}$ will have no entry on its bottom. Figure 12 illustrates $G_{2}$ and $G_{3}$.

There are now two cases to consider. First assume that there exists a critical point $C$ such that $C<A$ and $F(C)>F(B)$. Throughout the rest of the paper this shall be referred to as "case 1 ". In this case we set $\Gamma(A, B)=G_{n}$ where $n=|K(A, B)|$. Here, $|K(A, B)|$ denotes the cardinality of the set $K(A, B)$. For "case 2", when there does not exist a $C$ such that $C<A$ and $F(C)>F(B)$, we set $\Gamma(A, B)=G_{F(A, B)}$. That is, $\Gamma(A, B)$ is equal to the graph corresponding to the function $F(A, B)$.
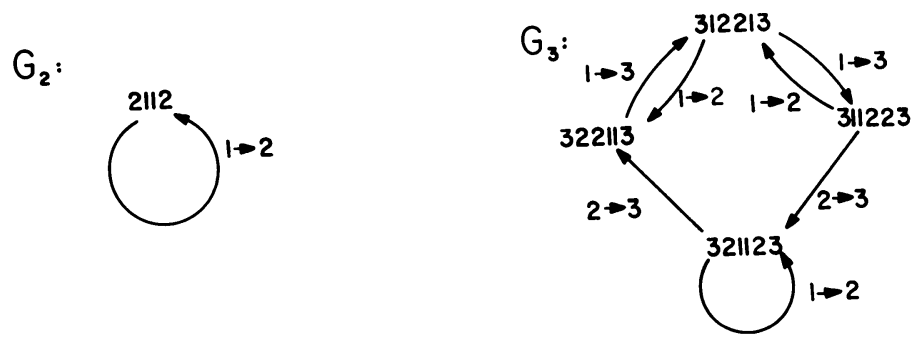

Figure 12 
Of course, the assumption $A<B$ is merely for convenience. If we had $A>B$, with $F(A)<F(B)$, we would still assume that there does not exist a critical point $C$ such that $B<C<A$ and $F(C)>F(B)$. There would still be the two cases to consider depending if there exists a $C$ such that $C>A$ and $F(C)>F(B)$.

We now state the main result of the paper.

THEOREM 5C.1. There exists an $L D G$ homomorphism $\Phi$ from $G_{F}$ onto $\Gamma(A, B)$. Furthermore, $\Phi\left(P_{F}\right)$ is an admissible path in $\Gamma(A, B)$.

(D) Some useful propositions. Throughout this section we assume that $F$ has $N$ critical points and $\mathscr{A}$ is the array

$$
\mathscr{A}=\frac{T_{1} T_{2} \cdots T_{m}}{B_{1} B_{2} \cdots B_{n}}
$$

Note that $m+n=2 N$. Sometimes it will be useful to rewrite $\mathscr{A}$ as

$$
\mathscr{A}=\frac{T_{1} T_{2} \cdots T_{m}}{T_{2 N} \cdots T_{m+2} T_{m+1}} .
$$

That is, $B_{i}=T_{j}$ where $j=2 N-i+1$ for $1 \leqslant i \leqslant n$.

Let $Y_{N}=\{0,1,2, \ldots, N\}$, and suppose that $S_{1}$ and $S_{2}$ are two, nonempty disjoint subsets of $Y_{N} \backslash\{0\}$. We say that $S_{1}$ interlaces $S_{2}$ in $\mathscr{A}$ if there exists integers $s_{1}, s_{2}, s_{3}$, $i, j, k$ and $l$ with $s_{1} \in S_{1}, s_{2} \in S_{2}, s_{3} \in S_{2}$ and $1 \leqslant i<j<k<l \leqslant 2 N$ such that either

$$
s_{1}=T_{i}, \quad s_{2}=T_{j}, \quad s_{1}=T_{k} \quad \text { and } \quad s_{3}=T_{l}
$$

or

$$
s_{2}=T_{i}, \quad s_{1}=T_{j}, \quad s_{3}=T_{k} \quad \text { and } \quad s_{1}=T_{l} .
$$

If there does not exist such integers we say that $S_{1}$ does not interlace $S_{2}$ in $\mathscr{A}$. Note that it is possible that $S_{1}$ interlaces $S_{2}$ in $\mathscr{A}$ while $S_{2}$ does not interlace $S_{1}$ in $\mathscr{A}$. For example, let

$$
\mathscr{A}=\frac{221}{133}, \quad S_{1}=\{1\} \quad \text { and } \quad S_{2}=\{2,3\} .
$$

The following result will be used often in the proof of Theorem 5C.1.

Proposition 5D.1. $\mathscr{C}(\mathscr{X})$ does not interlace $\mathscr{C}(A, B)$ in $\mathscr{A}$.

Proof. Suppose that the proposition is not true. Then there must exist critical points $C, D \in K(A, B)$ and $E \in \mathscr{X}$ such that $\left\{E^{*}\right\}$ interlaces $\left\{C^{*}, D^{*}\right\}$ in $\mathscr{A}$. We assume that there exist integers $i, j, k$ and $l$ such that $C^{*}=T_{i}, E^{*}=T_{j}, D^{*}=T_{k}$ and $E^{*}=T_{l}$. The other case, (5D.3a), is similar. We denote the trajectories which $T_{i}$, $T_{j}, T_{k}$ and $T_{l}$ correspond to as $C\left(z, \theta_{0}\right), E\left(z, \theta_{0}\right), D\left(z, \theta_{0}\right)$ and $\hat{E}\left(z, \theta_{0}\right)$, respectively. Let $\alpha(z)$ equal the union of the curves $E\left(z, \theta_{0}\right)$ and $\hat{E}\left(z, \theta_{0}\right)$, together with the point $\bar{E}$. Let $\beta(z)$ be the union of the curves $C\left(z, \theta_{0}\right)$ and $D\left(z, \theta_{0}\right)$, and the closed line interval connecting the points $\bar{C}$ and $\bar{D}$. The assumption that $\left\{E^{*}\right\}$ interlaces $\left\{C^{*}, D^{*}\right\}$ implies that $\alpha(z)$ and $\beta(z)$ must intersect. Since $F(U)<F(E)$ for $U \in(C, D),(1.6)$ implies that $\alpha(z)$ cannot intersect the line segment connecting $\bar{C}$ 
and $\bar{D}$. However, $\alpha(z)$ clearly cannot intersect $C\left(z, \theta_{0}\right)$ or $D\left(z, \theta_{0}\right)$, so the proof is complete.

The proof of the following proposition is quite similar to the one just given.

Proposition 5D.2. Let $S=\mathscr{C}(A, B) \backslash\{B\}$. Then $\{B\}$ does not interlace $S$ in $\mathscr{A}$.

Finally, the following result is of interest.

Proposition 5D.3. Assume that $C \in K(A)$ and $D \in \mathscr{Y}$. Then there cannot exist any $C \rightarrow D$ or $D \rightarrow C$ connections.

Proof. If $C \in K(A)$ and $D \in \mathscr{Y}$, then there exists a critical point $E$ which lies between $C$ and $D$ and satisfies $F(E)>\max \{F(C), F(D)\}$. In the proof of Proposition 2.3 we showed that this implies that there cannot exist any $C \rightarrow D$ or $D \rightarrow C$ connections.

(E) The map $\Phi$. In order to define $\Phi$ it is necessary to first introduce some more notation. We assume throughout that $F$ has $N$ critical points and $\mathscr{A}$ is the array given by (5D.1) or (5D.2). Recall that an array consists of a top and a bottom list of integers. Whenever we just write one list of integers it will always denote the top of the array, with the understanding that the bottom list is empty. Using the representation (5D.2) for $\mathscr{A}$ we set

$$
\beta(\mathscr{A})=T_{1} T_{2} \cdots T_{m} T_{m+1} T_{m+2} \cdots T_{2 N} .
$$

Recall that $Y_{N}=\{0,1,2, \ldots, N\}$. If $M$ is any subset of $Y_{N}$, we define $\hat{M} * \mathscr{A}$ to be the array obtained by deleting from $\mathscr{A}$ those elements not in $M$.

Suppose that $S$ is any set of $n$ distinct positive integers. Say $S=\left\{s_{1}, s_{2}, \ldots, s_{n}\right\}$. We denote by $\rho$ the unique function which maps $S$ onto $\{1, \ldots, n\}$ such that $\rho\left(s_{i}\right)<\rho\left(s_{j}\right)$ if and only if $s_{i}<s_{j}$. If $\mathscr{A}$ is the array given in (5D.1) we set

$$
\hat{\rho} * \mathscr{A}=\frac{\rho\left(T_{1}\right) \rho\left(T_{2}\right) \cdots \rho\left(T_{m}\right)}{\rho\left(B_{1}\right) \rho\left(B_{2}\right) \cdots \rho\left(B_{n}\right)} .
$$

We first consider the case when there exists a critical point $C$ such that $C<A$ and $F(C)>F(B)$. This was referred to as "case 1 " in the previous section. Recall that, in this case, $\Gamma(A, B)=G_{n}$ where $n$ is the cardinality of the set $K(A, B)$. The map $\Phi_{\theta}: Y_{N} \rightarrow Y_{n}$ is defined by

$$
\Phi_{\theta}(k)= \begin{cases}\rho(k) & \text { if } k \in \mathscr{C}(A, B), \\ 0 & \text { otherwise. }\end{cases}
$$

We define $\Phi(\mathscr{A})$ in two steps. First let

$$
\Phi_{1}(\mathscr{A})=\hat{\rho} * \hat{\mathscr{C}}(A, B) * \beta(\mathscr{A}) .
$$

Suppose that

$$
\Phi_{1}(\mathscr{A})=z_{1} z_{2} \cdots z_{2 n} \text {. }
$$

It follows from Proposition 5D.2 that either $z_{1}=z_{2 n}=n$, or there exists an integer $k$ such that $z_{k}=z_{k+1}=n$. If $z_{1}=z_{2 n}=n$, then let $\Lambda_{1}$ be the identity map and define $\Phi$ by

$$
\Phi(\mathscr{A}) \equiv \Lambda_{1} * \Phi_{1}(\mathscr{A}) \equiv \Phi_{1}(\mathscr{A})
$$


Otherwise, define $\Lambda_{1}$ and $\Phi$ by

$$
\Phi(\mathscr{A}) \equiv \Lambda_{1} * \Phi_{1}(\mathscr{A}) \equiv z_{k+1} z_{k+2} \cdots z_{2 n} z_{1} z_{2} \cdots z_{k}
$$

In either case, it will sometimes be convenient to reindex and write $\Phi(\mathscr{A})$ as

$$
\Phi(\mathscr{A})=\eta_{1} \eta_{2} \cdots \eta_{2 n} \text {. }
$$

Note that $\Phi(\mathscr{A}) \in G_{n}$.

It remains to define $\Phi$ on the edges. Suppose that $e \in E(\mathscr{A})$. If $e=\mathscr{A}$ we set $\Phi(e)=\Phi(\mathscr{A})$. So suppose that $e$ corresponds to a $C \rightarrow D$ connection. That is, $\Theta(e)=\left(C^{*}, D^{*}\right)$. If $C^{*} \notin \mathscr{C}(A, B)$ or $D^{*} \notin \mathscr{C}(A, B)$, we set $\Phi(e)=\Phi(\mathscr{A})$. If $\left\{C^{*}, D^{*}\right\} \subset \mathscr{C}(A, B)$ we claim that there exists an edge $f \in E(\Phi(\mathscr{A}))$ which corresponds to a $\Phi\left(C^{*}\right) \rightarrow \Phi\left(D^{*}\right)$ connection. To see why this is true, we use the representation (5D.2) for $\mathscr{A}$. There must exist an integer $j$ such that $C^{*}=T_{j}$ and $D^{*}=T_{j+1}$. Certainly the same is true in (5E.1). Since $\left\{C^{*}, D^{*}\right\} \subset \mathscr{C}(A, B), \hat{\mathscr{C}}(A, B)$ eliminates these integers of $\beta(\mathscr{A})$ not in $\mathscr{C}(A, B)$, and $\hat{\rho}$ simply renumbers the integers of the array, we find that there must exist an integer $l$ such that, in (5E.4), $z_{l}=\rho\left(C^{*}\right)=\Phi\left(C^{*}\right)$ and $z_{l+1}=\rho\left(D^{*}\right)=\Phi\left(D^{*}\right)$. Finally, recalling how (5E.6) was obtained from (5E.4) we find that there exists an integer $r$ such that $\eta_{r}=\Phi\left(C^{*}\right)$ and $\eta_{r+1}=\Phi\left(D^{*}\right)$. This implies what we claimed; that is, there exists an edge $f \in$ $E(\Phi(\mathscr{A}))$ which corresponds to a $\Phi\left(C^{*}\right) \rightarrow \Phi^{*}\left(D^{*}\right)$ connection. We now set $\Phi(e)$ $=f$.

We now consider case 2, assuming that there does not exist a critical point $C$ such that $C<A$ and $F(C)>F(B)$. Recall that in this case $\Gamma(A, B)=G_{F(A, B)}$. As before, we assume that the cardinality of the set $\mathscr{C}(A, B)$ is $n$. The map $\Phi_{\theta}: Y_{N} \rightarrow Y_{n}$ is again defined by (5E.2). Let $\Phi_{1}(\mathscr{A})$ be the array given by (5E.3) and (5E.4). We now show that there exists an integer $k$ such that $k<2 n$ and $z_{k}=z_{k+1}=n$. From Proposition 5D.2, the only other possibility is that $z_{1}=z_{2 n}=n$. We show that it is impossible for $z_{2 n}=n$. Choose $C$ so that $C<B$ and $F(C) \geqslant F(D)$ for all $D<B$. By assumption $F(C)<F(B)$. Note that $C \in K(A, B)$. Now (1.6) implies that $C_{S W}(z, \theta)$ never intersects the $U$-axis for all $\theta$. Since $C<B$, this implies that on the bottom of the array, there cannot be a $B^{*}$ to the left of the $C^{*}$ which corresponds to $C_{S W}(z, \theta)$, for any $\theta$. This means that in $\Phi_{1}(\mathscr{A})$, the $\Phi_{1}\left(C^{*}\right)$ which corresponds to $C_{S W}(z, \theta)$ lies to the right of both of the $\Phi_{1}\left(B^{*}\right)$. Since $\Phi_{1}\left(B^{*}\right)=n$, we obtain the desired conclusion that $z_{k}=z_{k+1}=n$ for some $k<2 n$. We now define $\Lambda_{2}$ and $\Phi$ by

$$
\Phi(\mathscr{A}) \equiv \Lambda_{2} * \Phi_{1}(\mathscr{A}) \equiv \frac{z_{1} z_{2} \cdots z_{k}}{z_{2 n} \cdots z_{k+2} z_{k+1}} .
$$

It remains to define $\Phi$ on the edges. Suppose that $e \in E^{*}(\mathscr{A})$. If $e=\mathscr{A}$ we let $\Phi(e)=(\Phi(\mathscr{A}))$. So suppose that $e$ corresponds to a $C \rightarrow D$ connection. If $C^{*} \notin$ $\mathscr{C}(A, B)$ or $D^{*} \notin \mathscr{C}(A, B)$, we let $\Phi(e)=\Phi(\mathscr{A})$. If $\left\{C^{*}, D^{*}\right\} \subset \mathscr{C}(A, B)$, a proof very similar to the one given for case 1 shows that there exists an edge $f \in E(\Phi(\mathscr{A})$ ) which corresponds to a $\Phi\left(C^{*}\right) \rightarrow \Phi\left(D^{*}\right)$ connection. We now set $\Phi(e)=f$.

(F) Proof that $\Phi$ is an $L D G$ homomorphism from $G_{F}$ into $\Gamma(A, B)$. The only condition of Definition 5B.1 which $\Phi$ does not trivially satisfy is (B). So suppose that 
$e \in E(\mathscr{A})$ corresponds to a $C \rightarrow D$ connection. We distinguish the two $C^{*}$ 's and two $D^{*}$ 's in $\mathscr{A}$ by $C_{1}, C_{2}, D_{1}$ and $D_{2}$, respectively. Assume that $e$ corresponds to a $C_{1} \rightarrow D_{1}$ connection.

We first consider the map $\beta$. Using the representation (5D.2) for $\mathscr{A}$ it follows that there exists an integer $k$ such that $C_{1}=T_{k}$ and $D_{1}=T_{k+1}$. We suppose that $D_{2}=T_{j}$. Clearly the same is true for $\beta(\mathscr{A})$. Hence, it makes sense to say that there is an edge $e_{1} \in E\left(\beta(\mathscr{A})\right.$ ) which corresponds to a $C_{1} \rightarrow D_{1}$ connection. We claim that

$$
\beta(e * \mathscr{A})=e_{1} * \beta(\mathscr{A}) .
$$

To prove this note that the only possible difference between $\beta(e * \mathscr{A})$ and $e_{1} * \beta(\mathscr{A})$ is the position of the $C_{1}$. This is because the only entry which changes when applying $e$ or $e_{1}$ is the $C_{1}$. However, in both cases $C_{1}$ is moved to the immediate right of the $D_{2}$.

We next consider the map $\hat{\mathscr{C}}(A, B)$. We shall consider the three cases:

$$
\begin{gathered}
C_{1} \notin \mathscr{C}(A, B), \\
C_{1} \in \mathscr{C}(A, B), \quad D_{1} \in \mathscr{C}(A, B), \\
C_{1} \in \mathscr{C}(A, B), \quad D_{1} \notin \mathscr{C}(A, B) .
\end{gathered}
$$

If $(5 \mathrm{~F} .2 \mathrm{a})$ is satisfied, we claim that

$$
\hat{\mathscr{C}}(A, B) * \beta(\mathscr{A})=\hat{\mathscr{C}}(A, B) * \beta(e * \mathscr{A}) .
$$

As before, we need only consider the position of $C_{1}$, because it is the only entry whose position changes. However, since $C_{1} \notin \mathscr{C}(A, B)$, it does not appear in either $\hat{\mathscr{C}}(A, B) * \beta(\mathscr{A})$ or $\hat{\mathscr{C}}(A, B) * \beta(e * \mathscr{A})$.

Now suppose that $(5 \mathrm{~F} .2 \mathrm{~b})$ is satisfied. Recall that in $\beta(\mathscr{A}), C_{1}$ is on the immediate left of $D_{1}$. The same is true in the array $\hat{\mathscr{C}}(A, B) * \beta(\mathscr{A})$ since $\left\{C_{1}, D_{1}\right\}$ $\subset \mathscr{C}(A, B)$. Hence, it makes sense to say that there is an edge $e_{2} \in$ $E(\hat{\mathscr{C}}(A, B) * \beta(\mathscr{A}))$ which corresponds to a $C_{1} \rightarrow D_{1}$ connection. Note that

$$
\hat{\mathscr{C}}(A, B) * \beta(e * \mathscr{A})=e_{2} * \hat{\mathscr{C}}(A, B) * \beta(\mathscr{A}) \text {. }
$$

This is because in both cases $C_{1}$ is on the immediate right of $D_{2}$.

Finally suppose that (5F.2c) is satisfied. Recall that $D_{1}=T_{k+1}$ and $D_{2}=T_{j}$. If $j<k+1$, then $\beta(\mathscr{A})$ has the form

$$
\beta(\mathscr{A})=\alpha D_{2} \gamma C_{1} D_{1} \delta .
$$

Here $\alpha, \gamma$ and $\delta$ are lists of integers. Note that

$$
\beta(e * \mathscr{A})=e *(\beta(\mathscr{A}))=\alpha D_{2} C_{1} \gamma D_{1} \delta
$$

Let $\alpha^{\prime}=\hat{\mathscr{C}}(A, B) * \alpha, \gamma^{\prime}=\hat{\mathscr{C}}(A, B) * \gamma$ and $\delta^{\prime}=\hat{\mathscr{C}}(A, B) * \delta$. From Proposition 5D.1 it follows that $\alpha^{\prime}$ and $\delta^{\prime}$ are empty. Since $C_{1} \in \mathscr{C}(A, B)$ and $D_{1} \notin \mathscr{C}(A, B)$ it follows that

$$
\hat{\mathscr{C}}(A, B) * \beta(\mathscr{A})=\gamma^{\prime} C_{1}
$$

while

$$
\hat{\mathscr{C}}(A, B) * \beta(e * \mathscr{A})=C_{1} \gamma^{\prime}
$$


If $k+1<j$, then $\beta(\mathscr{A})$ has the form

$$
\beta(\mathscr{A})=\alpha C_{1} D_{1} \gamma D_{1} \delta,
$$

where, as before, $\alpha, \gamma$ and $\delta$ are lists of integers. Then

$$
\beta(e * \mathscr{A})=e *(\beta(\mathscr{A}))=\alpha D_{1} \gamma D_{1} C_{1} \delta .
$$

Letting $\alpha^{\prime}, \gamma^{\prime}$ and $\delta^{\prime}$ be as before, we conclude from Proposition 5D.1 that $\gamma^{\prime}$ is empty. Hence,

$$
\hat{\mathscr{C}}(A, B) * \beta(\mathscr{A})=\alpha^{\prime} C_{1} \delta^{\prime}
$$

while

$$
\hat{\mathscr{C}}(A, B) * \beta(e * \mathscr{A})=\alpha^{\prime} C_{1} \delta .
$$

Note that if $k+1<j$, then $\hat{\mathscr{C}}(A, B) * \beta(\mathscr{A})=\hat{\mathscr{C}}(A, B) * \beta(e * \mathscr{A})$. This is not true if $j<k+1$.

We now consider the map $\hat{\rho}$. It is again necessary to consider the three cases shown in (5F.2). Recall that $\Phi_{1}(\mathscr{A}) \equiv \hat{\rho} * \hat{\mathscr{C}}(A, B) * \beta(\mathscr{A})$. If (5F.2a) is satisfied, then $(5 \mathrm{~F} .3 \mathrm{a})$ holds. Since $\rho$ is order preserving it is clear that in this case

$$
\Phi_{1}(\mathscr{A})=\Phi_{1}(e * \mathscr{A}) \text {. }
$$

Next suppose that (5F.2b) holds. Then $C_{1}$ is to the immediate left of $D_{1}$ in the array $\hat{\mathscr{C}}(A, B) * \beta(\mathscr{A})$. Therefore, $\rho\left(C_{1}\right)$ is to the immediate left of $\rho\left(D_{1}\right)$ in $\Phi_{1}(\mathscr{A})$. Hence there exists an edge $e_{3} \in E\left(\Phi_{1}(\mathscr{A})\right)$ which corresponds to a $\rho\left(C_{1}\right) \rightarrow \rho\left(D_{1}\right)$ connection. From (5F.3b) it follows that

$$
\Phi_{1}(e * \mathscr{A})=e_{3} * \Phi_{1}(\mathscr{A}) \text {. }
$$

Finally, suppose that (5F.2c) holds. Let $a=\hat{\rho}\left(\alpha^{\prime}\right), g=\hat{\rho}\left(\gamma^{\prime}\right)$ and $d=\hat{\rho}\left(\delta^{\prime}\right)$. If $j<k+1$, then (5F.6) implies that

$$
\Phi_{1}(\mathscr{A})=g \rho\left(C_{1}\right)
$$

while (5F.7) implies that

$$
\Phi_{1}(e * \mathscr{A})=\rho\left(C_{1}\right) g .
$$

If $j>k+1$, then (5F.10) and (5F.11) imply that

$$
\Phi_{1}(\mathscr{A})=\Phi_{1}(e * \mathscr{A})=a \rho\left(C_{1}\right) d .
$$

In order to complete the proof that $\Phi$ is an LDG homomorphism from $G_{F}$ into $\Gamma(A, B)$, we must consider the maps $\Lambda_{1}$ and $\Lambda_{2}$. We shall consider the three cases (5F.2a)-(5F.2c) separately. First suppose that (5F.2a) holds. Applying $\Lambda_{1}$ or $\Lambda_{2}$ to both sides of (5F.12) it follows that $\Phi(\mathscr{A})=\Phi(e * \mathscr{A})$. Since in this case $\Phi(e)=$ $\Phi(\mathscr{A})$, we obtain the desired result, $\Phi(e) * \Phi(\mathscr{A})=\Phi(e * \mathscr{A})$.

Next suppose that (5F.2b) holds. Then (5F.13) is true. To show that $\Phi(e * \mathscr{A})=$ $\Phi(e) * \Phi(\mathscr{A})$ we need only verify that $\rho\left(C_{1}\right)$ is in the same position in both arrays. For case $1, \rho\left(C_{1}\right)$ will be on the immediate right of $\rho\left(D_{2}\right)$ in both arrays. This is also true in case 2 if $\rho\left(D_{2}\right)$ is on the top side of $\Phi(e * \mathscr{A})$ or $\Phi(e) * \Phi(\mathscr{A})$. If $\rho\left(D_{2}\right)$ is on the bottom side of $\Phi(e * \mathscr{A})$ or $\Phi(e) * \Phi(\mathscr{A})$, then $\rho\left(C_{1}\right)$ will be on the immediate left of $\rho\left(D_{2}\right)$ in both arrays. 
Now, suppose that (5F.2c) holds. We first assume that $j>k+1$. Then (5F.16) holds. That is, $\Phi_{1}(\mathscr{A})=\Phi_{1}(e * \mathscr{A})$. Applying $\Lambda_{1}$ or $\Lambda_{2}$ to both sides of this identity we find that $\Phi(\mathscr{A})=\Phi(e * \mathscr{A})$. Since $\Phi(e)=\Phi(\mathscr{A})$ we conclude that $\Phi(e * \mathscr{A})=$ $\Phi(e) * \Phi(\mathscr{A})$.

Finally, suppose that (5F.2c) holds and $j<k+1$. We first consider case 1 . Suppose that $g=g_{1} g_{2} \cdots g_{r}$. The sentence between (5E.4) and (5E.5a) implies that either $g_{1}=\rho\left(C_{1}\right)=n$, or there exists an integer $i$ such that $g_{i}=g_{i+1}=n$. First assume the latter. Then (5F.14) and the definition of $\Lambda_{1}$ imply that

$$
\Phi(\mathscr{A})=g_{i+1} g_{i+2} \cdots g_{r} \rho\left(C_{1}\right) g_{1} \cdots g_{i} \text {. }
$$

Since $\Phi(e)=\Phi(\mathscr{A})$ we conclude that

$$
\Phi(e) * \Phi(\mathscr{A})=g_{i+1} \cdots g_{r} \rho\left(C_{1}\right) g_{1} \cdots g_{i} .
$$

Using (5F.15) we conclude that $\Phi(e * \mathscr{A})=\Phi(e) * \Phi(\mathscr{A})$. If $g_{1}=\rho\left(C_{1}\right)=n$, then (5F.14) and (5F.15) imply that

$$
\Phi(e * \mathscr{A})=\Phi(e) * \Phi(\mathscr{A})=n g_{2} g_{3} \cdots g_{r} n .
$$

To complete the proof that $\Phi$ is an LDG homomorphism from $G_{F}$ into $\Gamma(A, B)$ we show that it is impossible to have case 2 together with (5F.2c), $j<k+1$. To prove this, choose $E<B$ so that $F(E)>F(U)$ for all $U<B$. By definition, $E \in K(A, B)$. Note that $E_{S W}(z, \theta)$ can never cross the $U$-axis. This follows from (1.6) and the fact that $F(U)<F(E)$ for all $U<E$. Since we are in case 2 and $D \notin K(A, B)$, it follows that $D>B>E$. Therefore, if, for some $\theta, D_{N E}(z, \theta)$ or $D_{S W}(z, \theta)$ leaves through the bottom side of $\mathscr{R}$, it must necessarily cross to the right of $E_{S W}(z, \theta)$. Let $E_{1}$ be the entry in $\mathscr{A}$ which corresponds to $E_{S W}(z, \theta)$. We conclude that $E_{1} \in \delta$ where $\delta$ is as in (5F.4). If this were true, however, then (5F.4) would imply that $\left\{D^{*}\right\}$ interlaces $\left\{C^{*}, E^{*}\right\}$ in $\mathscr{A}$. That is, $\left\{D^{*}\right\}$ interlaces $\{\mathscr{C}(A, B)\}$ in $\mathscr{A}$. Proposition 5D.1 gives the desired contradiction.

(G) Proof that $\Phi$ is an $L D G$ homomorphism from $G_{F}$ onto $\Gamma(A, B)$. We begin by verifying Definition 5B.2(B). Let $y$ be a node in $\Gamma(A, B)$ and $f \in E(y)$. Assume that $\Phi(\mathscr{A})=y$. Furthermore, assume that $f$ corresponds to a $\hat{C} \rightarrow \hat{D}$ connection. Let $C^{*}=\Phi^{-1}(\hat{C})$ and $D^{*}=\Phi^{-1}(\hat{D})$. We consider a number of cases depending on the relationship between $C^{*}$ and $D^{*}$ in the array $\mathscr{A}$. We assume throughout that $\mathscr{A}$ is written as in (5D.2), with $C^{*}=T_{j}$ and $D^{*}=T_{k}$. The following six cases describe all the possible ways for $\Phi(\mathscr{A})=y$ :

(5G.1a) $j<k \leqslant m$, and $T_{i} \notin \mathscr{C}(A, B)$ for $j<i<k$,

(5G.1b) $\quad m+1 \leqslant j<k$, and $T_{i} \notin \mathscr{C}(A, B)$ for $j<i<k$,

(5G.1c) $j \leqslant m<k$, and $T_{i} \notin \mathscr{C}(A, B)$ for $j<i<k$,

(5G.1d) $k \leqslant m<j$, and $T_{i} \notin \mathscr{C}(A, B)$ if $1 \leqslant i<k$ or $j<i \leqslant 2 N$,

(5G.1e) $\quad k<j \leqslant m$, and $T_{i} \notin \mathscr{C}(A, B)$ if $j<i \leqslant 2 N$ or $1 \leqslant i<k$,

(5G.1f) $\quad m+1 \leqslant k<j$, and $T_{i} \notin \mathscr{C}(A, B)$ if $j<i \leqslant 2 N$ or $1 \leqslant i<k$.

Since two entries in $\mathscr{A}$ equal $C^{*}$ and two equal $D^{*}$, we assume that $T_{j}=C_{1}$ and $T_{k}=D_{1}$. This will allow us to distinguish the $C^{*}$ and $D^{*}$ of interest. 
Suppose that (5G.1a) holds. We wish to prove that there exists a finite sequence of connections, all of which are mapped by $\Phi$ to $y$, followed by a $C_{1} \rightarrow D_{1}$ connection. Of course, if $k:=j+1$, then there is nothing to prove, so we assume that $k>j+1$. Choose $l$ so that $j<l \leqslant k, C_{1}<T_{l}$ and $C_{1}>T_{i}$ for $j<i<l$. We assume that $l \neq k$. The other case will be treated shortly. Consider the sequence of connections

$$
T_{l-1} \rightarrow T_{l}, T_{l-2} \rightarrow T_{l}, \ldots, T_{j+1} \rightarrow T_{l} .
$$

Note that all of the edges corresponding to these connections are mapped to $y$. Now $T_{l}=T_{\rho}$ for some $\rho \neq l$. From the definition of $l$ it is clear that $\rho \notin(j, l)$. Hence, after the sequence of connections shown in (5G.2) we find that $C_{1}$ is on the immediate left of $T_{l}$. Since $T_{l} \notin \mathscr{C}(A, B)$, Proposition 5D.3 implies that $T_{l} \in \mathscr{C}(\mathscr{X})$. Proposition 5D.1 then implies that $\rho \in(l, k)$. We then take the $C_{1} \rightarrow T_{l}$ connection. After this connection $C_{1}$ is still to the left of $D_{1}$. There is still no element of $\mathscr{C}(A, B)$ between $C_{1}$ and $D_{1}$. However, the number of integers which lie between $C_{1}$ and $D_{1}$ has decreased by at least two. Continuing this process we eventually arrive at an array of the form

$$
\frac{\cdots C_{1} \alpha_{1} \alpha_{2} \cdots \alpha_{s} D_{1} \cdots}{\cdots}
$$

The dots denote integers in the array. Each $\alpha_{i}$ satisfies $\alpha_{i}<C_{1}$. We then take the sequence of connections

$$
\alpha_{s} \rightarrow D_{1}, \alpha_{s-1} \rightarrow D_{1}, \ldots, \alpha_{1} \rightarrow D_{1} .
$$

We can then take the desired $C_{1} \rightarrow D_{1}$ connection.

The proof for case (5G.1b) is quite similar to the one just given. Now assume that (5G.1c) is satisfied. Let $E$ be the critical point which satisfies $E \geqslant B$ and $F(E)>$ $F(U)$ for all $U>B$. Then $E_{N E}(z, \theta)$ never crosses the $U$-axis for any value of $\theta$. This, together with Proposition 5D.1 implies that there exist integers $r$ and $s$ such that $E^{*}=T_{r}=T_{s}, j<r \leqslant m$ and $m+1 \leqslant s<k$. The proof now proceeds as in the preceding cases. The existence of $E$ guarantees that eventually we can push $C_{1}$ to the bottom side of the array, after which we are in the situation of case (5G.1b).

Next assume that (5G.1d) holds. For case one, when there exists a critical point $\hat{C}$ with $\hat{C}<A$ and $F(\hat{C})>F(A)$, the proof is quite similar to the one just given. One proves that there exists a critical point $E$ and integers $r$ and $s$ such that $E<A$, $F(E)>F(B), E^{*}=T_{r}=T_{s}, j<r \leqslant 2 N$ and $1 \leqslant s<k$. We are then able to push $C_{1}$ to the top of the array so we are in the situation of (5G.1a).

Now assume that (5G.1d) holds and we are in case two. Choose $r$ and $s$ so that $r<s$ and $T_{r}=T_{s}=B^{*}$. From Proposition 5D.2 it follows that $k \leqslant r<s<j$. If this is true, however, it is impossible for $\Phi\left(C_{1}\right)$ and $\Phi\left(D_{1}\right)$ to be adjacent integers in $y$. Hence, (5G.1d) is impossible for case two.

A similar proof shows that (5G.1e) and (5G.1f) are impossible for case two. The proof for case one is similar to the preceding proofs. If (5G.1e) holds for case one, then one shows there exists critical points $E_{1}$ and $E_{2}$, and integers $r_{1}, s_{1}, r_{2}$ and $s_{2}$ such that $E_{1}^{*}=T_{r_{1}}=T_{s_{1}}, E_{2}^{*}=T_{r_{2}}=T_{s_{2}}, E_{1} \geqslant B, E_{2}<A, E_{1}^{*} \geqslant B^{*}, E_{2}^{*} \geqslant B^{*}$, $j<r_{1} \leqslant m, m+1 \leqslant s_{1}<s_{2} \leqslant 2 N$ and $1 \leqslant r_{2}<k$. This implies that we can push 
$C_{1}$ in a clockwise fashion around the array until we are in the situation described by (5G.1a). A similar analysis works if (5G.1f) holds.

To complete the proof that $\Phi$ is an LDG homomorphism from $G_{F}$ onto $\Gamma(A, B)$ we show that a generator of $G_{F}$ is mapped to a generator of $\Gamma(A, B)$. We may then apply Lemma 5B.2. There is no problem for case two, when $\Gamma(A, B)=G_{F}(A, B)$. For case one, $\Gamma(A, B)=G_{n}$. We prove that every node in $G_{n}$ is a generator for $G_{n}$. This is done by induction on $n$. The result is clearly true for $n=2$, since there is only one node in $G_{2}$. Now assume the result is true for $G_{n-1}$, and let $\mathscr{A}$ and $\mathscr{B}$ be two arrays in $G_{n}$. We must prove that there exists a path from $\mathscr{A}$ to $\mathscr{B}$. Suppose that

$$
\mathscr{A}=n A_{1} A_{2} \cdots A_{2 n-2} n \text { and } \mathscr{B}=n B_{1} B_{2} \cdots B_{2 n-2} n .
$$

A proof similar to the proof of Proposition 5D.1 shows that $\{n-1\}$ does not interlace $\{1, \ldots, n-2\}$ in either $\mathscr{A}$ or $\mathscr{B}$. Hence, either $A_{1}=A_{2 n-2}=n-1$, or there exists an integer $j$ such that $A_{j}=A_{j+1}=n-1$. Similarly, either $B_{1}=B_{2 n-2}=n-$ 1 , or there exists an integer $k$ such that $B_{k}=B_{k+1}=n-1$. Suppose that there exists an integer $j$ such that $A_{j}=A_{j+1}=n-1$. Consider the path $P_{1}$ consisting of the series of connections

$$
A_{2 n-2} \rightarrow n, A_{2 n-3} \rightarrow n, \ldots, A_{j+2} \rightarrow n, A_{j+1} \rightarrow n .
$$

Then $P_{1} * \mathscr{A}$ has the form

$$
P_{1} * \mathscr{A}=n n-1 \cdots n-1 n .
$$

If $A_{1}=A_{2 n-2}=n-1$ we set $P_{1} * \mathscr{A}=\mathscr{A}$. If $B_{1}=B_{2 n-2}=n-1$ we use induction to conclude that there exists a path $P_{2}$ such that $P_{2} *\left(P_{1} * \mathscr{A}\right)=\mathscr{B}$. If not, we use induction to conclude that there exists a path $P_{2}$ such that

$$
P_{2} *\left(P_{1} * \mathscr{A}\right)=n n-1 B_{k+2} B_{k+3} \cdots B_{2 n-2} B_{1} B_{2} \cdots B_{k-1} n-1 n .
$$

Now let $P_{3}$ be the path consisting of the connections

$$
n-1 \rightarrow n, B_{k-1} \rightarrow n, B_{k-2} \rightarrow n, \ldots, B_{1} \rightarrow n .
$$

We then have $P_{3} *\left(P_{2} *\left(P_{1} * \mathscr{A}\right)\right)=\mathscr{B}$.

(H) Proof that $\Phi\left(P_{F}\right)$ is an admissible path in $\Gamma(A, B)$. We use the same notation as in the previous section. That is, $y$ is a node in $\Gamma(A, B), f \in E(y)$, and $\Phi(\mathscr{A})=y$. Furthermore, $f$ corresponds to a $\hat{C} \rightarrow \hat{D}$ connection. Let $C^{*}=\Phi^{-1}(\hat{C})$ and $D^{*}=$ $\Phi^{-1}(\hat{D})$. As before, $\mathscr{A}$ is given by (5D.2). We assume that $C^{*}=T_{j}$ and $D^{*}=T_{k}$. Of course, two entries in $\mathscr{A}$ equal $C^{*}$ and two equal $D^{*}$. Here $T_{j}$ and $T_{k}$ are equal to the entries which are mapped to the $\hat{C}$ and $\hat{D}$ of the $\hat{C} \rightarrow \hat{D}$ connection. Assume that $\mathscr{A}$ is the array associated with the phase plane determined by (1.3) with speed $\theta_{0}$. We must show that there exists a $C \rightarrow D$ connection for some speed $\theta<\theta_{0}$. The proof is broken up into the six cases shown in (5G.1a)-(5G.1f).

First assume that $(5 \mathrm{G} .1 \mathrm{a})$ holds. The proof is quite similar to the proof of Proposition 2.1 given in subsection 1(D). Let $P_{c}$ and $P_{d}$ be the points on the boundary of $\mathscr{R}$ corresponding to $T_{j}$ and $T_{k}$. We denote the trajectories on which $P_{c}$ 
and $P_{d}$ lie by $C(z)$ and $D(z)$, respectively. As in the proof of Proposition 2.1 we consider the eight cases:

$$
\begin{aligned}
& C<D, \quad C(z)=C_{N E}\left(z, \theta_{0}\right), \quad D(z)=D_{N E}\left(z, \theta_{0}\right), \\
& \text { (5H.1b) } \quad C<D, \quad C(z)=C_{S W}\left(z, \theta_{0}\right), \quad D(z)=D_{N E}\left(z, \theta_{0}\right) \text {, } \\
& \text { (5H.1c) } \quad C<D, \quad C(z)=C_{N E}\left(z, \theta_{0}\right), \quad D(z)=D_{S W}\left(z, \theta_{0}\right) \text {, } \\
& \text { (5H.1d) } \quad C<D, \quad C(z)=C_{S W}\left(z, \theta_{0}\right), \quad D(z)=D_{S W}\left(z, \theta_{0}\right) \text {, } \\
& \text { (5H.1e) } \quad C>D, \quad C(z)=C_{N E}\left(z, \theta_{0}\right), \quad D(z)=D_{N E}\left(z, \theta_{0}\right) \text {, } \\
& \text { (5H.1f) } \quad C>D, \quad C(z)=C_{S W}\left(z, \theta_{0}\right), \quad D(z)=D_{N E}\left(z, \theta_{0}\right) \text {, } \\
& \text { (5H.1g) } \quad C>D, \quad C(z)=C_{N E}\left(z, \theta_{0}\right), \quad D(z)=D_{S W}\left(z, \theta_{0}\right) \text {, } \\
& \text { (5H.1h) } \quad C>D, \quad C(z)=C_{S W}\left(z, \theta_{0}\right), \quad D(z)=D_{S W}\left(z, \theta_{0}\right) \text {. }
\end{aligned}
$$

For the time being we assume that (5H.1a) holds. As in the proof of Proposition 2.1, we follow $C(z)$ and $D(z)$ backwards from $P_{c}$ and $P_{d}$ and find that there exists a verticle line $l$, given by $U=P$, for some $P$, and numbers $\gamma_{c}$ and $\gamma_{d}$ such that:

$$
\begin{gathered}
\gamma_{c}>\gamma_{d}>0, \\
C(z) \text { crosses } l \text { at }\left(P, \gamma_{c}\right), \\
D_{N W}\left(z, \theta_{0}\right) \text { crosses } l \text { at }\left(P, \gamma_{d}\right),
\end{gathered}
$$

and

$$
C(z) \text { and } D_{N W}\left(z, \theta_{0}\right) \text { do not cross } l
$$

at any other value between $\gamma_{c}$ and $\gamma_{d}$.

For $\theta>0$, let $C(z, \theta)=C_{N E}(z, \theta), D(z, \theta)=D_{N E}(z, \theta)$ and $\hat{D}(z, \theta)=D_{N W}(z, \theta)$. For $\theta$ sufficiently close to $\theta_{0}$ we define functions $\gamma_{c}(\theta)$ and $\gamma_{d}(\theta)$ to be the $V$ coordinate of the places where $C(z, \theta)$ and $\hat{D}(z, \theta)$ cross $l$. Proposition 2.1 was proved by showing that if $\theta_{1}$ is chosen so that $\theta_{1}<\theta_{0}$ and no $C \rightarrow D$ connections exist for $\theta \in\left(\theta_{1}, \theta_{0}\right)$, then $\gamma_{c}(\theta)$ and $\gamma_{d}(\theta)$ can be chosen to be continuous functions for $\theta \in\left(\theta_{1}, \theta_{0}\right)$. Unfortunately, this may not be true now. We claim, however, that if no $C \rightarrow D$ connection exists for $\theta \in\left(\theta_{1}, \theta_{0}\right)$, then, for $\theta \in\left(\theta_{1}, \theta_{0}\right), \gamma_{c}(\theta)$ and $\gamma_{d}(\theta)$ can be chosen so that $(5 \mathrm{H} .2 \mathrm{a})-(5 \mathrm{H} .2 \mathrm{c})$ hold with $\gamma_{c}$ replaced by $\gamma_{c}(\theta)$ and $\gamma_{d}$ replaced by $\gamma_{d}(\theta)$.

Assume throughout that no $C \rightarrow D$ connection exists for $\theta \in\left(\theta_{1}, \theta_{0}\right)$. There is no problem in showing that $\gamma_{d}(\theta)$ is continuous in $\left(\theta_{1}, \theta_{0}\right)$. Before discussing what may happen to $\gamma_{c}(\theta)$ we introduce some more notation. Suppose, for the moment, that $\gamma_{c}(\theta)$ is continuous for $\theta \in\left(\theta_{1}, \theta_{0}\right)$. For $\theta \in\left(\theta_{1}, \theta_{0}\right)$ choose $z_{1}(\theta), z_{2}(\theta), z_{3}(\theta)$ and $z_{4}(\theta)$ so that

$$
\begin{gathered}
C\left(z_{1}(\theta), \theta\right)=\left(P, \gamma_{c}(\theta)\right), \quad C\left(z_{2}(\theta), \theta\right)=P_{c}, \\
\hat{D}\left(z_{3}(\theta), \theta\right)=\left(P, \gamma_{d}(\theta)\right) \text { and } D\left(z_{4}(\theta), \theta\right)=P_{d} .
\end{gathered}
$$


Here $P_{c}$ and $P_{d}$ denote the points on the boundary of $P$ where $C(z, \theta)$ and $D(z, \theta)$ first leave $\mathscr{R}$. Let $\hat{l}(\theta)$ be the portion of $l$ with $\gamma_{c}(\theta) \leqslant V \leqslant \gamma_{d}(\theta)$. Let $K(\theta)$ be the union of the curves

$$
\begin{aligned}
& C(z(\theta), \theta) \text { for } z_{1}(\theta) \leqslant z \leqslant z_{2}(\theta), \\
& \hat{D}(z(\theta), \theta) \text { for } z_{3}(\theta) \leqslant z<\infty, \\
& D(z(\theta), \theta) \text { for }-\infty<z \leqslant z_{4}(\theta), \text { and } \\
& \hat{l}(\theta) .
\end{aligned}
$$

Then $K(\theta)$ divides $\mathscr{R}$ into two parts which we denote by $R_{1}(\theta)$ and $R_{2}(\theta)$. Let $B_{1}(\theta)$ and $B_{2}(\theta)$ be the portions of the boundary of $\mathscr{R}$ which lie in the closure of $R_{1}(\theta)$ and $R_{2}(\theta)$, respectively. We assume that $R_{1}(\theta)$ is the region which satisfies the property that a trajectory which crosses $\hat{l}(\theta)$ must thereafter remain in $R_{1}(\theta)$ until it crosses $B_{1}(\theta)$. Note that the converse is also true. That is, if a trajectory leaves $\mathscr{R}$ through $B_{1}(\theta)$, then it must have crossed $\hat{l}(\theta)$.

Now assume that $\gamma_{c}(\theta)$ is continuous for $\theta \in\left(\theta_{1}, \theta_{0}\right)$, but discontinuous at $\theta=\theta_{1}$. Then there exists a $C \rightarrow E$ connection with speed $\theta_{1}$ for some critical point $E$. Of course, this connection involves $C_{N E}\left(z, \theta_{1}\right)$, as opposed to $C_{S W}\left(z, \theta_{1}\right)$. Let $\gamma_{c}\left(\theta_{1}\right)=$ $\lim _{\theta \rightarrow \theta_{1}} \gamma_{c}(\theta)$. From Lemma 3E.1 it follows that this limit exists. Since $\gamma_{c}(\theta)$ is discontinuous at $\theta=\theta_{1},\left(P, \gamma_{c}\left(\theta_{1}\right)\right)$ lies on either $E_{N E}\left(z, \theta_{1}\right)$ or $E_{S W}\left(z, \theta_{1}\right)$, as opposed to $E_{N W}\left(z, \theta_{1}\right)$ or $E_{S E}\left(z, \theta_{1}\right)$. Assume that the unstable trajectory on which $\left(P, \gamma_{c}\left(\theta_{1}\right)\right)$ lies is $E_{S W}\left(z, \theta_{1}\right)$. The other case is similar. From the proof of Proposition 2.2, we conclude that there exists a $\delta>0$ such that, for $\theta_{1}<\theta<\theta_{1}+\delta$, $E_{S W}(z, \theta)$ crosses $\hat{l}(\theta)$. Hence, $E_{S W}(z, \theta)$ leaves $\mathscr{R}$ through $B_{1}(\theta)$. From the proof of Proposition 2.3 we conclude that for $\theta$ less than, but close to $\theta_{1}, C(z, \theta)$ lies as close as we wish to $E_{N E}(z, \theta)$. We claim that for $\theta$ greater than, but close to $\theta_{1}, E_{N E}(z, \theta)$ crosses $\hat{l}(\theta)$.

The first step in proving this is to show that $E^{*}>D^{*}$. Note that $E^{*}>C^{*}$. This is because there exists a $C \rightarrow E$ connection. Suppose that $E^{*}<D^{*}$. Choose $\theta_{2}$ equal to the supremum of all values of $\theta$ for which $\theta<\theta_{0}$, and there exists a critical point $M$ which satisfies

(a) $C^{*}<M^{*}<D^{*}$, and

(b) either $M_{N E}(z, \theta)$ or $M_{S W}(z, \theta)$ leaves $\mathscr{R}$ through $B_{1}(\theta)$.

By assumptions, $\theta_{1}<\theta_{2}<\theta_{0}$. Since $\theta_{2} \neq \theta_{0}$, there must exist an $M \rightarrow Q$ connection with speed $\theta_{2}$ for some other critical point $Q$. Now there exists a $\delta_{1}>0$ such that, for $\theta_{2}<\theta<\theta_{2}+\delta_{1}$, one of the unstable trajectories from $M$, say $M(z, \theta)$, leaves $\mathscr{R}$ through $B_{2}$, while for $\theta_{2}-\delta_{1}<\theta<\theta_{2}, M(z, \theta)$ leaves $\mathscr{R}$ through $B_{1}$. Propositions 2.2 and 2.3 imply that, for $\theta=\theta_{2}$, one of the unstable trajectories from $Q$ leaves $\mathscr{R}$ through $B_{1}$, while the other leaves $\mathscr{R}$ through $B_{2}$. Hence $\left\{Q^{*}\right\}$ interlaces $\left\{C^{*}, D^{*}\right\}$ for the array with speed $\theta_{2}$. From Proposition 5D.1 we conclude that $C^{*}<Q^{*}<D^{*}$. This, however, contradicts the way $\theta_{2}$ and $M$ were chosen.

Having shown that $E^{*}>D^{*}$ and $E_{S W}(z, \theta)$ leaves $\mathscr{R}$ through $B_{1}$ for $\theta \in\left(\theta_{1}, \theta_{1}+\right.$ $\delta$ ), we can apply Proposition 5D.1 to conclude that $E_{N E}(z, \theta)$ leaves $\mathscr{R}$ through $B_{1}$ for $\theta \in\left(\theta_{1}, \theta_{1}+\delta\right)$. Therefore, $E_{N E}(z, \theta)$ crosses $\hat{l}(\theta)$ for $\theta \in\left(\theta_{1}, \theta_{1}+\delta\right)$. By choosing $\delta$ sufficiently small we may assume that $C(z, \theta)$ is as close as we please to 
$E_{N E}\left(z, \theta_{1}\right)$ for $\theta_{1}-\delta<\theta<\theta_{1}$. Therefore, $C(z, \theta)$ must cross $\hat{l}\left(\theta_{1}\right)$ for $\theta_{1}-\delta<\theta$ $<\theta_{1}$. That is, $\gamma_{c}(\theta)$ can be chosen so that $\gamma_{c}(\theta)>\gamma_{d}(\theta)$ for $\theta_{1}-\delta<\theta<\theta_{1}$. This completes the proof that if no $C \rightarrow D$ connection exists for $\theta \in\left(\theta_{1}, \theta_{0}\right)$, then $\gamma_{c}(\theta)$ and $\gamma_{d}(\theta)$ can be chosen so that (5H.2) is satisfied with $\gamma_{c}$ and $\gamma_{d}$ replaced by $\gamma_{c}(\theta)$ and $\gamma_{d}(\theta)$, respectively.

What we have shown is that, in some sense, as long as no $C \rightarrow D$ connection exists, $C_{N E}(z, \theta)$ lies above $D_{N W}(z, \theta)$. However, using the fact that $C^{*}<D^{*}$, we find that $C_{N E}(z, 0)$ lies below $D_{N E}(z, 0)$. This shooting argument completes the proof of the theorem for the case when (5G.1a) and (5H.1a) hold.

Still assuming that (5G.1a) holds, we only say a few words about the proofs for the other cases shown in $(5 \mathrm{H} .1)$. The proof for cases $(5 \mathrm{H} .1 \mathrm{~b}),(5 \mathrm{H} .1 \mathrm{~g})$ and $(5 \mathrm{H} .1 \mathrm{~h})$ are quite similar to the one just given. Just as in the proof of Proposition 2.1, one shows that cases $(5 \mathrm{H} .1 \mathrm{c})-(5 \mathrm{H} .1 \mathrm{f})$ are impossible.

We must still consider the other cases shown in (5G.1a)-(5G.1f). However, the proofs for these cases are really no different than the one just given. The reason is that the main assumption used about the array $\mathscr{A}$ in the preceding proof is that if we start at $T_{j}$ and move in the clockwise direction around the array until we reach $T_{k}$, then every integer we pass is not in $\mathscr{C}(A, B)$. This is true for every case shown in (5G.1a)-(5G.1f).

6. Conclusions. We now demonstrate how the directed graphs are used to determine how many connections exist between two fixed critical points. Given $A$ and $B$, with $F(A)<F(B)$, the results discuss whether there must exist zero, exactly one, a finite number, or an infinite number of $A \rightarrow B$ connections. One trivial result, which was proved earlier, is that if there exists a critical point $C$ which lies between $A$ and $B$, and $F(C)>F(B)$, then there cannot exist any $A \rightarrow B$ connections. So we assume throughout this section that such a critical point $C$ does not exist. For convenience we assume that $A<B$.

We first assume that there does exist a critical point $D$ such that $D<A$ and $F(D)>F(B)$. This was referred to as case one earlier. Recall that in this case $\Gamma(A, B)=G_{n}$, where $n=|K(A, B)|$. We prove that if $n=2$ or $n=3$, then there must exist an infinite number of $A \rightarrow B$ connections.

If $n=2$, then $\Gamma(A, B)=G_{2}$ which is shown in Figure 12. According to Theorem 5C.1 there exists an LDG homomorphism from $G_{F}$ onto $G_{2}$. Note that $\Phi\left(A^{*}\right)=1$ and $\Phi\left(B^{*}\right)=2$. To prove that there exists an infinite number of $A \rightarrow B$ connections, it suffices to prove that $\Phi\left(P_{F}\right)$, the image of the path corresponding to $F$, contains an infinite number of $1 \rightarrow 2$ connections. This is clear, however, because $G_{2}$ consists of only one node and one edge which begins and ends at that node. Because $\Phi\left(P_{F}\right)$ is admissible, it is forced to keep going around the loop an infinite number of times.

Now suppose that $n=3$. Suppose that $K(A, B)=\{A, C, B\}$. Then there exists a graph homomorphism $\Phi$ from $G_{F}$ onto $G_{3}$ such that $\Phi\left(A^{*}\right)=1, \Phi\left(C^{*}\right)=2$ and $\Phi\left(B^{*}\right)=3$. It follows that there exists an infinite number of $A \rightarrow B$ connections, if we can show that the path $\Phi\left(P_{F}\right)$ contains an infinite number of $1 \rightarrow 3$ connections. Since $|K(C, B)|=2, \Phi\left(P_{F}\right)$ must contain an infinite number of $2 \rightarrow 3$ connections. 
Considering Figure 12 this implies that $\Phi\left(P_{F}\right)$ must be at the node 321123 or the node 322113 an infinite number of times. If $\Phi\left(P_{F}\right)$ is at the node 321123 , then, because $\Phi\left(P_{F}\right)$ is admissible and a $2 \rightarrow 3$ edge branches from $321123, \Phi\left(P_{F}\right)$ must be at 322113 an infinite number of times. Since a $1 \rightarrow 3$ connection branches from 322113 , we conclude that $\Phi\left(P_{F}\right)$ contains an infinite number of $1 \rightarrow 3$ connections, the desired result.

It is natural to ask how many $A \rightarrow B$ connections exist when $\Gamma(A, B)=G_{n}$ for $n>3$. We do not know the answer to this question for the following reason. Suppose that $n=4$. In (6.1) we give an example of a subpath in $G_{4}$ which does not contain any $1 \rightarrow 4$ connections:

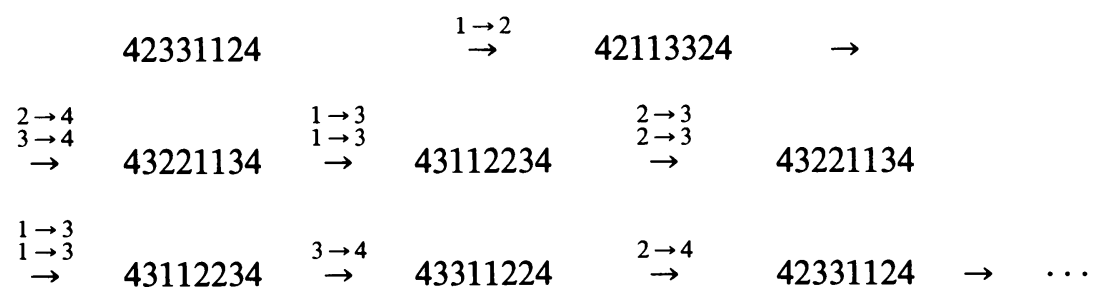

Note that the first and last arrays shown are the same. If we keep repeating the subpath shown, then the resulting path contains an infinite number of $3 \rightarrow 4,2 \rightarrow 4$, $2 \rightarrow 3,1 \rightarrow 3$ and $1 \rightarrow 2$ connections. By writing out the entire graph $G_{4}$, we find that (6.1) gives an admissible path in $G_{4}$. This seems to imply that there may not exist any $A \rightarrow B$ connections if $\Gamma(A, B)=G_{4}$. However, we do not know if there actually is a function $F$ such that the path corresponding to $F$ is the one shown in (6.1). Note that it is also possible, in fact easier, to construct admissible paths in $G_{4}$ for which there exists an infinite number of $1 \rightarrow 4$ connections.

We now assume that if $C<A$, then $F(C)<F(B)$. We first show that if there does not exist $C$ such that $A<C<B$ and $F(C)>F(A)$, then there must exist at least one $A \rightarrow B$ connection. Suppose that $\eta$ is the generator of $G_{F}$, and

$$
\Phi(\eta)=\frac{T_{1} T_{2} \cdots T_{n}}{B_{1} B_{2} \cdots B_{n}}
$$

The assumption that there does not exist a $C$ such that $A<C<B$ and $F(C)>F(A)$ implies that there exists an integer $k$ such that $\Phi\left(A^{*}\right)=T_{k}$ and $\Phi\left(B^{*}\right)=T_{k+1}$. Since $\Phi\left(P_{F}\right)$ is admissible it must contain a $T_{k} \rightarrow T_{k+1}$ connection. Hence, $P_{F}$ must contain an $A \rightarrow B$ connection. If $F(D)<F(A)$ for all $D<A$, then the $A \rightarrow B$ connection is unique. This is because $\Gamma(A, B)$ is the graph:

$$
\begin{aligned}
& \frac{12}{12} \\
& \downarrow 1 \rightarrow 2 \\
& \frac{2}{112}
\end{aligned}
$$


If there does exist $C$ such that $A<C<B$ and $F(C)>F(A)$, then there may or may not exist an $A \rightarrow B$ connection. For example, suppose $F$ is as shown in Figure 13(A). The graph corresponding to $F$ is shown in Figure 13(B). From the result just proved we conclude that there must exist unique $1 \rightarrow 2$ and $2 \rightarrow 3$ connections. The graph corresponding to $F$ demonstrates that there exists a $1 \rightarrow 3$ connection if and only if the speed of the $1 \rightarrow 2$ connection is less than the speed of the $2 \rightarrow 3$ connection.

If there does not exist a critical point $D$ such that $D<A$ and $F(D)>F(A)$, then, as we now prove, there can exist at most one $A \rightarrow B$ connection. The assumption that $F(U)<F(B)$ for all $U<B$, and (1.6) implies that $B_{S W}(z, \theta)$ never crosses the $U$-axis. It always leaves $\mathscr{R}$ through its bottom side. Similarly, $A_{S W}(z, \theta)$ always leaves $\mathscr{R}$ through its bottom side, never crossing the $U$-axis. Suppose that the array for some value of $\theta$ is given by $\mathscr{A}(\theta)$. We also assume that an $A \rightarrow B$ connection exists with speed $\theta_{0}$. For $\theta_{1}$ a bit smaller than $\theta_{0}, \mathscr{A}(\theta)$ has the form

$$
\mathscr{A}\left(\theta_{1}\right)=\frac{\cdots}{\cdots A_{1} \cdots A_{2} B_{1} \cdots} \text {. }
$$

Here $A_{1}$ corresponds to $A_{S W}\left(z, \theta_{1}\right), A_{2}$ corresponds to $A_{N E}\left(z, \theta_{1}\right)$ and $B_{1}$ corresponds to $B_{S W}\left(z, \theta_{1}\right)$. If we let $\beta$ be the map defined in the previous section and let $B_{2}$ correspond to $B_{N E}\left(z, \theta_{1}\right)$, then $\beta * \mathscr{A}\left(\theta_{1}\right)$ has the form

$$
\beta * \mathscr{A}\left(\theta_{1}\right)=\cdots B_{2} \cdots B_{1} A_{2} \cdots A_{1} \cdots \text {. }
$$

Note that, for all $\theta, A_{1}$ always lies to the right of $B_{2}, B_{1}$ and $A_{2}$ in $\beta * \mathscr{A}(\theta)$.

We wish to show that there cannot exist another $A \rightarrow B$ connection. This is only possible if $A_{1}$ is on the immediate left of a $B$ in $\beta * \mathscr{A}(\theta)$ for some $\theta<\theta_{1}$. The only way $A_{1}$ can get to the left of one of the $B$ 's is if there exists a $D$ such that, for some $\theta<\theta_{0}, \beta * \mathscr{A}(\theta)$ has the form

(a) $\cdots D^{*} B_{2} \cdots B_{1} \cdots A_{2} D^{*} \cdots A_{1} \cdots$, or

(b) $\cdots B_{2} \cdots D^{*} B_{1} \cdots A_{2} D^{*} \cdots A_{1} \cdots$.

In either case, $\left\{D^{*}\right\}$ interlaces $\left\{A^{*}\right\}$. The proof of Proposition 5D.1 shows that this is impossible.

Now suppose that $F(C)<F(B)$ for all $C<B$, but there does exist a critical point $D<A$ such that $F(D)>F(A)$. Then there may exist a finite number of $A \rightarrow B$ connections. This is illustrated in Figure 6 where one sees that there may exist a finite number of $1 \rightarrow 3$ connections. To prove that there cannot exist an infinite number of $A \rightarrow B$ connections we note that there must exist a $D \rightarrow B$ connection for
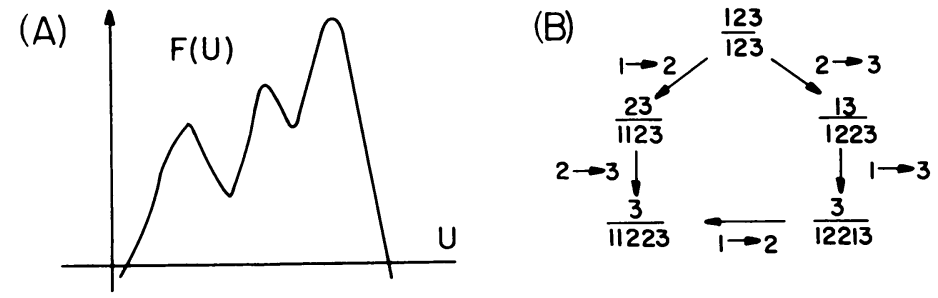

FIGURE 13 
some positive speed, say $\theta=\theta_{0}$. It follows from Proposition 2.4 that there can exist at most a finite number of $A \rightarrow B$ connections with speed greater than $\theta_{0}$. A proof very similar to the one given in the preceding paragraph implies that there cannot exist any $A \rightarrow B$ connections with speed less than $\theta_{0}$. Hence, there can exist at most a finite number of $A \rightarrow B$ connections.

AcKnowledgement. I wish to thank Charles Conley and Glen Richard Hall for their encouragement and stimulating discussions.

\section{REFERENCES}

1. D. G. Aronson and H. F. Weinberger, Nonlinear diffusion in population genetics, combustion and nerve propagation, Proc. Tulane Program in Partial Differential Equations and Related Topics, Lecture Notes in Math., No. 466, Springer-Verlag, Berlin, 1975, pp. 5-49.

2. P. C. Fife and J. B. McLeod, The approach of solutions of nonlinear diffusion equations to traveling front solutions, Arch. Rational Mech. Anal. 65 (1975), 335-361; Bull. Amer. Math. Soc. 81 (1975), 1075-1078.

3. P. S. Hagan, Traveling wave and multiple traveling wave solutions of parabolic equations, SIAM J. Math. Anal. 13 (1982), 717-738.

4. D. Terman, Traveling wave solutions of a multistable reaction-diffusion system, Nonlinear Partial Differential Equations (J. Smoller, ed.), Contemp. Math., Vol. 17, Amer. Math. Soc., Providence, R.I., 1982, pp. 361-378.

Department of Mathematics, University of Wisconsin, Madison, Wisconsin 53706

Current address: Michigan State University, Department of Mathematics, East Lansing, Michigan 48824 